JOURNAL OF THE

AMERICAN MATHEMATICAL SOCIETY

Volume 22, Number 3, July 2009, Pages 859-888

S 0894-0347(08)00620-6

Article electronically published on October 27, 2008

\title{
BETTI NUMBERS OF GRADED MODULES AND COHOMOLOGY OF VECTOR BUNDLES
}

\author{
DAVID EISENBUD AND FRANK-OLAF SCHREYER
}

Dedicated to Mark Green, whose work connecting Algebraic Geometry and Free Resolutions has inspired us for a quarter of a century, on the occasion of his sixtieth birthday

\section{INTRODUCTION}

The Betti table of a graded module $M$ over a graded ring $S$ is numerical data consisting of the minimal number of generators in each degree required for each syzygy module of $M$. In their remarkable paper [2006, Mats Boij and Jonas Söderberg conjectured that the Betti table of a Cohen-Macaulay module over a polynomial ring is a positive linear combination of Betti tables of modules with pure resolutions. We prove a strengthened form of their conjectures. Applications include a proof of the Multiplicity Conjecture of Huneke and Srinivasan and a proof of the convexity of a fan naturally associated to the Young lattice.

Similarly, the cohomology table of a coherent sheaf $\mathcal{F}$ on a projective variety is numerical data of the dimension of each cohomology group of each twist of the sheaf. With the same tools we show that the cohomology table of any vector bundle on projective space is a positive rational linear combination of the cohomology tables of what we call supernatural vector bundles. Using this result we give new bounds on the slope of a vector bundle in terms of its cohomology.

Our results show that the pure Betti tables and the cohomology tables of supernatural vector bundles generate the extremal rays in the appropriate convex rational cones of Betti tables and cohomology tables. Though these cones are not dual to one another in the usual sense, we use certain supernatural bundles to define the supporting hyperplanes of the cone of Betti tables, and certain pure resolutions to define the supporting hyperplanes of the cone of cohomology tables.

After the first version of this paper was posted, Boij and Soederberg 2008 showed that the functionals we define here actually cut out the rational cone generated by all Betti tables - not just those of Cohen-Macaulay modules, as in this paper. Using this, they prove strong versions of the Mulitplicity Conjecture for all modules.

We next describe the results more precisely. Throughout this paper we will work with the polynomial ring $S=K\left[x_{1}, \ldots, x_{n}\right]$ over a fixed, arbitrary field, and with the projective space $\mathbb{P}^{n-1}:=\mathbb{P}_{K}^{n-1}$ over that field. To simplify notation we often write $m:=n-1$. All modules will be finitely generated graded $S$-modules with maps homogeneous of degree zero. All sheaves on $\mathbb{P}^{m}$ will be coherent.

Received by the editors January 17, 2008.

2000 Mathematics Subject Classification. Primary 14F05, 13D02; Secondary 13D25, 14N99.

(C)2008 American Mathematical Society Reverts to public domain 28 years from publication 
Betti tables. Let $\mathbb{B}$ be the rational vector space of rational Betti tables: that is, $\mathbb{B}=\bigoplus_{-\infty}^{\infty} \mathbb{Q}^{n+1}$, which we think of as the space of tables of rational numbers with $n+1$ columns and rows numbered by the integers. To any length $n$ complex of finitely generated free $S$-modules

$$
F: \bigoplus_{j} S(-j)^{\beta_{0, j}} \leftarrow \cdots \leftarrow \bigoplus_{j} S(-j)^{\beta_{i, j}} \leftarrow \cdots \leftarrow \bigoplus_{j} S(-j)^{\beta_{n, j}} \leftarrow 0
$$

we associate the Betti table $\beta(F) \in \mathbb{B}$ whose entry in the $i$-th column and $j$-th row is $\beta_{i, i+j}$ :

\begin{tabular}{c|cccc}
$j \backslash i$ & 0 & 1 & $\cdots$ & $n$ \\
\hline$\vdots$ & $\vdots$ & $\vdots$ & $\cdots$ & $\vdots$ \\
0 & $\beta_{0,0}$ & $\beta_{1,1}$ & $\cdots$ & $\beta_{n, n}$ \\
1 & $\beta_{0,1}$ & $\beta_{1,2}$ & $\cdots$ & $\beta_{n, n+1}$ \\
$\vdots$ & $\vdots$ & $\vdots$ & $\cdots$ & $\vdots$
\end{tabular}

Finally, to any graded $S$-module $M$ we associate the Betti table $\beta(M)$ of its minimal free resolution. Note that the direct sum of modules or resolutions corresponds to addition of Betti tables.

By a degree sequence (of length $c$ ) we will mean a strictly increasing sequence of integers $d=\left(d_{0}<\cdots<d_{c}\right)$. The resolution $F$ is called pure, with degree sequence $d$, if $\beta_{i, j}=0$ except when $j=d_{i}$. In this case Herzog and Kühl [1984] show that

$$
\beta_{i, d_{i}}=\lambda \prod_{j \neq i} \frac{1}{\left|d_{j}-d_{i}\right|} \quad \text { for } 0 \leq i \leq c
$$

for some rational number $\lambda$. The proof relies on the equations imposed on the $\beta_{i, j}(M)$ by the vanishing of the first $c$ coefficients of the Hilbert polynomial of $M$, corresponding to the fact that the support of $M$ has codimension $c$. We will call these the Herzog-Kühl equations.

The first of the Boij-Söderberg Conjectures is the existence of Cohen-Macaulay modules with pure resolutions having any given degree sequence. They prove this in the case $n=2$. It was proved for all $n$ in characteristic zero by Eisenbud-Fl $\varnothing y s t a d-$ Weyman [2007. We prove it in general.

Theorem 0.1. Every pure Betti table corresponding to a degree sequence of length $c \leq n$ is a rational multiple of the Betti table of the minimal free resolution of a Cohen-Macaulay $S$-module.

We give the termwise partial order to the set of sequences of a given length,

$$
\left(d_{0}<\cdots<d_{c}\right) \leq\left(d_{0}^{\prime}<\cdots<d_{c}^{\prime}\right) \quad \Leftrightarrow \quad d_{i} \leq d_{i}^{\prime} \text { for all } i .
$$

A totally ordered subset of a partially ordered set is called a chain. The other parts of the Boij-Söderberg Conjectures may be summarized as follows.

Theorem 0.2. The Betti table of any finitely generated graded Cohen-Macaulay $S$-module of codimension c can be expressed uniquely as a positive rational linear combination of the Betti tables of codimension c Cohen-Macaulay modules with pure resolutions whose degree sequences form a chain.

We strengthen this result to show that all Betti tables of the minimal resolutions of all finitely generated graded $S$-modules, whether Cohen-Macaulay or not, lie inside a certain rational cone. 
As noted in Boij-Söderberg [2006], the Multiplicity Conjecture of Huneke and Srinivasan (see Herzog and Srinivasan [1998) follows from the conclusion of Theorem 0.2. Indeed, the motivation of Boij and Söderberg in formulating their bold and striking conjectures was apparently to prove the Multiplicity Conjecture. In doing so, it seems to us, they provided a remarkable new way of approaching free resolutions.

We denote by $e(M)$ the multiplicity of a module $M$; if $M$ is of finite length, a case to which all this reduces, then $e(M)=\operatorname{dim}_{K}(M)$, the vector space dimension.

Corollary 0.3 (Huneke-Srinivasan Multiplicity Conjecture). If $M=S / I$ is a Cohen-Macaulay algebra of codimension $c$, then

$$
\frac{1}{c !} \prod_{i} \min \left\{j \mid \beta_{i, j} \neq 0\right\} \leq e(M) \leq \frac{1}{c !} \prod_{i} \max \left\{j \mid \beta_{i, j} \neq 0\right\} .
$$

One can include all Cohen-Macaulay modules generated in degree zero if one replaces $e(M)$ by a normalized multiplicity $e(M) / \beta_{0,0}(M)$. See Francisco-Srinivasan 2007. for a recent survey of the many papers on this conjecture and its generalizations. For another application of Theorems 0.2 and 0.1 see Söderberg [2006].

To prove Theorem 0.2 we construct the supporting hyperplanes of the cone of positive rational multiples of Betti tables of Cohen-Macaulay modules. These are defined in terms of certain free complexes that come, in turn, from supernatural vector bundles, which are vector bundles with special cohomology tables.

Cohomology tables. Let $\mathbb{B}^{*}=\prod_{-\infty}^{\infty} \mathbb{Q}^{n+1}$ be the dual of $\mathbb{B}$. We think of $\mathbb{B}^{*}$ as the space of rational cohomology tables, tables with $n+1$ rows and columns numbered by the integers, defined as follows: to any complex of graded free $S$-modules

$$
E: \quad 0 \rightarrow E^{0} \rightarrow E^{1} \rightarrow \cdots \rightarrow E^{n} \rightarrow 0
$$

we write $\gamma_{i, d}(E)$ for the value of the Hilbert function of $H^{i}(E)$ in degree $d$.

For example, let $\mathcal{E}$ be a vector bundle on $\mathbb{P}^{n-1}$, and write $\mathcal{E}^{*}$ for the dual bundle $\mathcal{H o m}\left(\mathcal{E}, \mathcal{O}_{\mathbb{P}^{n-1}}\right)$. If $E$ is the dual of a free resolution of the graded module $\bigoplus_{d} H^{0}\left(\mathcal{E}^{*}(d)\right)$, then $\gamma_{i, d}=h^{i}(\mathcal{E}(d))$ for $i<n-1$ and $\gamma_{i, d}=0$ for $i=n-1$ or $n$. A modification of these examples, with carefully chosen vector bundles, will be used to define the supporting hyperplanes needed for the proof of Theorem 0.2 see Proposition 6.8.

In either case, the associated cohomology table is the table in $\mathbb{B}^{*}$ with $\gamma_{i, d-i}$ in the $i$-th row and the $d$-th column:

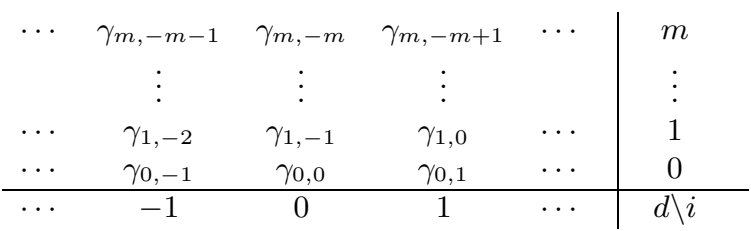

We make this choice of indexing so that the cohomology table of a vector bundle $\mathcal{E}$ coincides with the Betti table of the Tate resolution of $\mathcal{E}$. This is a minimal, doubly infinite exact free complex over the exterior algebra on $n$ generators that is connected with Bernstein-Gel'fand-Gel'fand duality. It is studied in EisenbudFløystad-Schreyer 2003] and Eisenbud-Schreyer 2003]. For consistency with the notation of that paper, we number the rows from the bottom and the columns from left to right as in the table above. Proposition 6.3 gives some general restrictions on cohomology tables. 
Following Hartshorne-Hirschowitz [1982] we say that a sheaf $\mathcal{F}$ on $\mathbb{P}^{n-1}=\mathbb{P}_{K}^{n-1}$ has natural cohomology if, for each integer $d$, the cohomology $H^{i}(\mathcal{F}(d))$ is non-zero for at most one value of $i$. We will say that $\mathcal{F}$ has supernatural cohomology if, in addition, the Hilbert polynomial $\chi(\mathcal{F}(d))$ has distinct integral roots. In this case we define the root sequence of $\mathcal{F}$ to be the sequence of roots in decreasing order, $z_{1}>\cdots>z_{m}$. If $m=n-1$, the case of primary interest to us, then any sheaf with natural cohomology is locally free (Remark 6.5), so we will generally speak of supernatural vector bundles.

Theorem 0.4. Any strictly decreasing sequence of $n-1$ integers is the root sequence of a supernatural vector bundle on $\mathbb{P}^{n-1}$.

In characteristic zero one can also construct supernatural bundles with every root sequence using Bott's vanishing theorem. This was explained to us by Jerzy Weyman. The construction, made explicit in Eisenbud-Schreyer 2003, Theorem 5.6 , and summarized below, is to apply an appropriate Schur functor to the universal rank $n-1$ quotient bundle on $\mathbb{P}^{n-1}$.

The condition of supernatural cohomology is very tight: the cohomology table of a supernatural sheaf is determined by its root sequence and its rank (see Theorem 6.4). For example, the cohomology table of a supernatural rank 3 vector bundle on $\mathbb{P}^{3}$ with root sequence $z=(3,-1,-4)$ must be

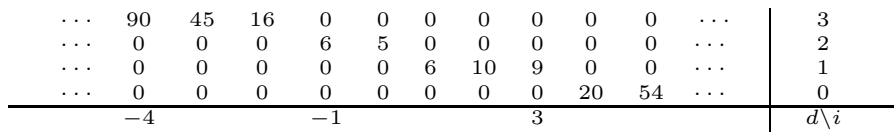

Inside the rational vector space $\mathbb{B}^{*}$ lies the set of actual cohomology tables of vector bundles, and since the direct sum of bundles corresponds to the addition of tables, it is natural to consider the rational cone that this set generates. We will show in 8 that this convex cone is the union of the simplicial cones of the fan of supernatural bundles. The cones in this fan correspond to termwise totally ordered sets of root sequences and have as extremal rays the cohomology tables of supernatural bundles. Moreover the supporting hyperplanes of the cone of cohomology tables of vector bundles are given by linear functionals defined from pure free resolutions of modules of finite length, using the same construction as we use for the proof of the Boij-Söderberg Conjectures.

Theorem 0.5. The cohomology table of any vector bundle on $\mathbb{P}_{K}^{n-1}$ has a unique expression as a positive rational linear combination of the supernatural cohomology tables corresponding to a chain of root sequences.

As a corollary, we obtain an analogue of the Multiplicity Conjecture in the vector bundle setting, giving new bounds for the slope of a vector bundle in terms of its cohomology. To state it, we say that the cohomology range of a vector bundle $\mathcal{E}$ on $\mathbb{P}^{m}$ is a pair of weakly decreasing sequences of integers

$$
r(\mathcal{E})=\left(r_{1} \geq \cdots \geq r_{m+1}=-\infty\right)<R(\mathcal{E})=\left(\infty=R_{0} \geq \cdots \geq R_{m}\right)
$$

that can be characterized as the termwise greatest and least weakly decreasing sequences, respectively, for which $H^{i} \mathcal{E}(d-i) \neq 0 \Rightarrow r_{i+1}<d<R_{i}$ (see $\S 7$ for another characterization). 
Corollary 0.6. If $\mathcal{E}$ is a vector bundle on $\mathbb{P}^{m}$, then the slope $\mu(\mathcal{E}):=\operatorname{deg} \mathcal{E} / \operatorname{rank} \mathcal{E}$ satisfies

$$
\frac{-1}{m} \sum_{i=1}^{m} R_{i}(\mathcal{E}) \leq \mu(\mathcal{E}) \leq \frac{-1}{m} \sum_{i=1}^{m} r_{i}(\mathcal{E}) .
$$

Bilinear functionals of resolutions and complexes. The heart of this paper is the analysis of certain bilinear forms on $\mathbb{B} \times \mathbb{B}^{*}$ and their positivity and vanishing properties. The central object is the form

$$
\langle B, C\rangle:=\sum_{\{i, j, k \mid j \leq i\}}(-1)^{i-j} \beta_{i, k} \gamma_{j,-k},
$$

where $B=\left(\beta_{i, i+d}\right)$ is a Betti table and $C=\left(\gamma_{j, d-j}\right)$ is a cohomology table. In $₫ 3$ we prove that when $C$ is the cohomology table of a free complex $E$ and $B$ is the Betti table of a free resolution $F$ over $S$, then

$$
\langle B, C\rangle=\sum_{j} \chi\left(F_{\geq j} \otimes H^{j}(E)\right) \geq 0 .
$$

Here $\chi$ denotes the Euler characteristic of the degree 0 part of the complex; that is,

$$
\chi\left(F_{\geq j} \otimes H^{j}(E)\right):=\sum_{k \geq j}(-1)^{k-j} \operatorname{dim}_{K}\left(\left(F_{k} \otimes H^{j}(E)\right)_{0}\right) .
$$

The functionals $\langle F, E\rangle=:\langle\beta(F), \gamma(E)\rangle$ do not directly give the supporting hyperplanes we need for the proof of Theorem 0.2 . because the cone of minimal resolutions of Cohen-Macaulay modules does not contain all non-minimal resolutions. In $\$ 4$ we construct a family of modifications $\langle B, C\rangle_{c, \tau}$ that are still non-negative when $B$ is the Betti table of a minimal free resolution. Applied with $E$ a supernatural vector bundle, or $F$ a pure free resolution, these define the functionals needed for the proofs of Theorems 0.2 and 0.5 .

The set of Betti tables. Though Theorem 0.2 gives the rational cone of minimal free resolutions of Cohen-Macaulay modules, it does not tell us which points in $\mathbb{B}$ are in the monoid of Betti tables of actual resolutions. Eisenbud, Fløystad and Weyman [2007] conjecture that any sufficiently large integral point on a ray of the cone corresponding to a pure resolution should be a $\beta(M)$ (this is not true in general for rays not corresponding to pure resolutions). Daniel Erman 2007] gives many additional restrictions that points in the rational cone satisfy if they come from resolutions, and shows that if $d<D$, then the monoid of resolutions of Cohen-Macaulay modules generated in degree $\geq d$ and having regularity $\leq D$ is finitely generated. Of course one can ask similar questions about cohomology tables.

The monoid of resolutions differs in characteristic zero and in finite characteristic. For example, take $n=5$ and let $M$ be a general Artinian Gorenstein factor ring of $S$ with Hilbert function $1,5,5,1$. Such rings can be obtained from the homogeneous coordinate rings of canonical curves of genus 7 by factoring out a regular sequence of linear forms. In characteristic zero the general ring of this type has Betti table

$$
\beta(M)=\begin{array}{cccccc}
1 & 0 & 0 & 0 & 0 & 0 \\
0 & 10 & 16 & 0 & 0 & 0 \\
0 & 0 & 0 & 16 & 10 & 0 \\
0 & 0 & 0 & 0 & 0 & 1
\end{array}
$$


as proven by Schreyer [1986]. But in characteristic 2 Kunte 2008] shows that no Cohen-Macaulay module has this Betti table. (Modules of finite length with twice this Betti table are easily constructed experimentally.) The Betti table of the general canonical curve of genus 7 in characteristic 2 was shown by Schreyer [1986] (see also Mukai [1992] and 2003]) to be

$$
\beta(M)=\begin{array}{cccccc}
1 & 0 & 0 & 0 & 0 & 0 \\
0 & 10 & 16 & 1 & 0 & 0 \\
0 & 0 & 1 & 16 & 10 & 0 \\
0 & 0 & 0 & 0 & 0 & 1
\end{array}
$$

The structure of this paper. In $\$ 1$ we give the algorithm for producing the decomposition of Theorem 0.2 suggested by Boij and Söderberg, and some examples of how it can be used. In $\$ 2$ we review the construction of the Boij-Söderberg fan associated to pure Betti tables. In $\$ 2$ we review the description of the exterior facets of this fan discovered by Boij-Söderberg [2006], and we explain an algorithmic construction of the supporting hyperplane of an exterior facet.

Sections 3 and 4 describe the bilinear forms that we use, together with their positivity and vanishing properties.

Our analysis of sheaves with supernatural cohomology is carried out in 8 , and the proof of Theorem 0.2 is completed in 97 . The proofs of Theorem 0.5 and Corollary 0.6 are carried out in 8 .

\section{An algorithm And an example: Consecutive Betti numbers}

The correctness of the following algorithm for decomposing a Betti table may clarify the meaning of Theorem 0.2 , from which it follows easily. It was conjectured in Boij-Söderberg [2006].

Decomposition Algorithm. Input: A graded Cohen-Macaulay $S$-module $M$ of codimension $c$.

Output: A list of positive rational coefficients $r_{i}$ and pure Betti Tables $\alpha^{(i)}$ whose degree sequences form a chain, such that $\beta=\sum r_{i} \alpha^{(i)}$.

1. BEGIN: Set $L$ equal to the empty list. Set $\beta:=\beta(M)$.

2. For $i=0, \ldots, c$ let $d_{i}=\min \left\{j \mid \beta_{i, j} \neq 0\right\}$. Let $\alpha$ be a pure Betti table with degree sequence $d=\left(d_{0}, \ldots, d_{c}\right)$. Let $r$ be the largest rational number such that $\beta^{\prime}:=\beta-r \alpha$ has non-negative entries.

3. Add $(r, \alpha)$ to the list $L$. If $\beta^{\prime}=0$, then END. Otherwise, set $\beta:=\beta^{\prime}$, and go to step 2 .

Here are some examples showing how Theorem 0.2 gives bounds on consecutive Betti numbers and limits the extent of "non-cancellation" in minimal resolutions.

Example 1.1. Let $B_{x}$ be the Betti table

$$
B_{x}=\begin{array}{cccccc}
1 & 0 & 0 & 0 & 0 & 0 \\
0 & 10 & 16 & x & 0 & 0 \\
0 & 0 & x & 16 & 10 & 0 \\
0 & 0 & 0 & 0 & 0 & 1
\end{array}
$$

In characteristic zero, the homogeneous coordinate ring of the general canonical curve of genus 7 has resolution with Betti table $B_{0}$, and it is known (Schreyer [1986]) that if the curve admits a realization as a plane sextic with 3 nodes, then the resolution has Betti table $B_{9}$. The Decomposition Algorithm above begins with a Betti table

$$
B^{\prime}=\begin{array}{cccccc}
5 & 0 & 0 & 0 & 0 & 0 \\
0 & 60 & 128 & 90 & 0 & 0 \\
0 & 0 & 0 & 0 & 20 & 0 \\
0 & 0 & 0 & 0 & 0 & 3
\end{array}
$$


that corresponds to the pure degree sequence $0,2,3,4,6,8$. This implies that if $B_{x}$ is a rational multiple of the Betti table of a Cohen-Macaulay module, then $x / 16 \leq 90 / 128$, that is $x \leq 11.25$, and Theorem 0.1 shows that there is a module of finite length whose Betti table is a multiple of $B_{11}$. In fact, writing $B^{\prime \prime}$ for the Betti table of the resolution dual to the one described by $B^{\prime}$, the reader may check that

$$
B_{11}=\frac{1}{45} B_{0}+\frac{11}{90} B^{\prime}+\frac{11}{90} B^{\prime \prime} .
$$

We do not know whether there are actually modules of finite length having Betti table $B_{11}$.

We can apply Theorem 0.5 to get a sharp bound on the linear strand of a resolution. Using Boij-Söderberg [2008], one could even drop the Cohen-Macaulay condition, which is why we have written "projective dimension" instead of "codimension".

Corollary 1.2. Let $K$ be any field and let $M$ be a graded Cohen-Macaulay module of projective dimension $\leq c$, generated in degree $\geq 0$ over $K\left[x_{1}, \ldots, x_{n}\right]$. If $\beta_{p+1, p+1}(M)=0$, then

$$
\beta_{p, p}(M) \leq \frac{c+2-p}{2 p} \beta_{p-1, p-1}(M) .
$$

Proof. Consider, a module with pure resolution having degree sequence $d=\left(d_{0}, \ldots\right.$, $\left.d_{c}\right)$, and the ratio of its Betti numbers $\beta_{p-1, d_{p-1}} / \beta_{p, d_{p}}$. One checks from the Herzog-Kühl formula that this ratio is a monotonically increasing function of the partial degree sequence $\left(d_{p+1}, \ldots, d_{c}\right)$. The ratio given in the corollary is the one associated to the pure resolution with the smallest degree sequence having $d_{0}=0, d_{p}=p, d_{p+1}>p+1$, namely, $0,1, \ldots, p, p+2, \ldots, c+1$.

\section{The BoIJ-SÖDERBERg FAN AND ITS FACETS}

A central insight of Boij and Söderberg [2006] is the identification of a certain simplicial fan inside $\mathbb{B}$. We begin by describing it.

For each interval $a<b$ of degree sequences, we consider the order complex of this interval whose simplices are the totally ordered subsets (chains) in the interval. For example, if $a_{0}<b_{0}$ are integers, then the interval between $\left(a_{0}, a_{0}+1, \ldots, a_{0}+n\right)$ and $\left(b_{0}, b_{0}+1, \ldots, b_{0}+n\right)$ is the well-known Young poset associated to the Schubert cell decomposition of the Grassmannian $G\left(n+1, b_{0}-a_{0}\right)$. Since every maximal chain of degree sequences between $d$ and $e$ has $1+\sum\left(d_{i}-e_{i}\right)$ elements, the order complex is equidimensional. In fact, the restriction to particular intervals $[a, b]$ in the Young poset is unnecessary, since the fan in a larger interval restricts to the fans in smaller intervals.

Boij and Söderberg show that the Betti tables coming from any one chain of degree sequences are linearly independent and that the map taking a degree sequence to a sequence of Betti numbers of the corresponding pure resolution provides a geometric realization of the order complex as a simplicial fan - that is, a collection of simplicial cones intersecting along faces - in $\mathbb{B}$. We will call this fan the BoijSöderberg fan. They conjectured that the union of the cones in the Boij-Söderberg fan is the convex cone of Betti tables of Cohen-Macaulay modules, and this is the content of Theorems 0.1 and 0.2 
We will call a facet of a simplicial cone in the Boij-Söderberg fan "exterior" if it is contained in a unique simplicial cone in the fan. The exterior facets are described as follows.

Proposition 2.1 (Boij-Söderberg [2006, Proposition 2.12]). Fix an interval $[\underline{d}, \bar{d}]$ in the poset of degree sequences, let $\Delta$ be a maximal chain of degree sequences between $\underline{d}$ and $\bar{d}$, and let $\Phi=\Delta \backslash\{f\}$ be a facet of $\Delta$. The facet $\Phi$ is exterior iff one of the following holds:

1. $\Phi$ is obtained from $\Delta$ by removing the minimal or maximal element. In the former case the supporting hyperplane of $\Phi$ is $\beta_{i, \underline{d}_{i}}=0$, where $i$ is the index where $\underline{d}$ and $\underline{d}^{+}$, the next sequence in the chain, differ, and dually in the latter case; or

2. the degree sequences $f^{-}$and $f^{+}$immediately below and above $f$ in $\Delta$ differ in exactly one position, say $f_{k}^{-}<f_{k}<f_{k}^{+}$. Since $\Delta$ is maximal we must have $f_{k}^{-}=f_{k}-1, f_{k}^{+}=f_{k}+1$, and the equation of the supporting hyperplane is $\beta_{k, f_{k}}=0$; or

3. the degree sequences $f^{-}$and $f^{+}$immediately below and above $f$ in $\Delta$ differ in exactly two adjacent positions, say $\tau$ and $\tau+1$, and we have $f_{\tau}=f_{\tau}^{-}=$ $f_{\tau}^{+}-1, f_{\tau+1}=f_{\tau+1}^{-}+1=f_{\tau+1}^{+}$and $f_{\tau+1}=f_{\tau}+2$ (see Example 2.4 for a picture).

We denote the facet described in part 3 by facet $(f, \tau)$. To show that one of these exterior facets of the Boij-Söderberg fan is an exterior facet of the cone of Betti tables, it suffices to prove that a supporting hyperplane of $\Phi$ is positive on all Betti tables of minimal free resolutions of modules of finite length. Without the existence this condition implies only that the cone of Betti tables is contained in the fan. The form of the equations for the supporting hyperplanes of the exterior facets in parts 1 and 2 of Proposition 2.1 makes this positivity obvious, so it suffices to treat facets of the form facet $(f, \tau)$ as in part 3 of the Proposition.

Since the Boij-Söderberg fan lies in a proper subspace of $\mathbb{B}$, the facet equation for a given facet is not unique - we may add any combination of the Herzog-Kühl equations. However, we will show that there are special linear functions defining the facets that are non-negative on the Betti table of any minimal free resolution. The individual $\beta_{i, j}$ satisfy this property for facets of types 1 and 2 in Proposition 2.1. We will exhibit distinguished equations for the supporting hyperplane of any facet of the form facet $(f, \tau)$, which we will call the upper and lower equations. We will show that the upper equation is non-negative on every minimal free resolution. It can be constructed algorithmically:

Proposition 2.2. Let $f^{-}<f<f^{+}$be degree sequences as in part 3 of Proposition 2.1. There is a unique hyperplane $U$ in $\mathbb{B}$ that contains facet $(f, \tau)$ and also all tables concentrated in degrees $\geq f^{+}$, which we call the upper hyperplane for facet $(f, \tau)$. Similarly, there is a unique lower hyperplane that contains all tables $\leq f^{-}$.

We will call the equation of the upper hyperplane the upper equation of the facet.

Proof. Proposition 2.12 of Boij-Söderberg [2006] shows that the only thing that really matters about facet $(f, \tau)$ is the pair of degree sequences $f^{-}, f^{+}$, in the sense that within the linear space defined by the Herzog-Kühl equations, the same linear functionals vanish on all facets not containing $f$ but containing $f^{-}, f^{+}$. Thus facet $(f, \tau)$ contains all pure Betti diagrams coming from degree sequences $\leq f^{-}$or 
$\geq f^{+}$in the partial order. Using this we can construct the equation of the upper hyperplane inductively as follows.

We identify linear functions on $\mathbb{B}$ with (possibly infinite) tables of integers with $n+1$ columns (see Example 2.4). Choose a maximal chain in $\mathbb{B}$ containing $f^{-}, f, f^{+}$. Using this chain, we will construct the table corresponding to the linear function vanishing on the facet, which we call the facet equation. We begin by putting zeros in all the entries of the table corresponding to degree sequences that are $\geq f^{+}$; that is, we put a zero in column $i$ and each row with index $\geq f_{i}^{+}-i$ for each $i=0 \ldots n$. Suppose that the Betti numbers corresponding to $f^{-}$are $\beta_{0}, \ldots, \beta_{n}$. In column $\tau$, row $f_{\tau}^{-}-\tau$ we put $\beta_{\tau+1}$ and in column $\tau+1$, row $f_{\tau+1}^{-}-(\tau+1)$ we put $-\beta_{\tau}$; this ensures that our functional will vanish on the Betti table corresponding to $f^{-}$as well as on the one corresponding to $f^{+}$and will be positive on that corresponding to $f$.

Whatever we put in the other entries of the table, this functional will vanish on every pure Betti table with degree sequence $d \geq f^{+}$. We can now solve for the remaining (infinitely many) coefficients inductively. We start with $f^{-}$, and at each stage we choose the next smaller degree sequence in our chain. The corresponding Betti table contains only one non-zero entry in the region that is not yet determined, so the vanishing condition allows us to solve uniquely for the corresponding element of the table we are constructing.

The same idea could be used with the roles of $f^{-}$and $f^{+}$reversed to produce the equation of the lower hyperplane.

The entries in rows $\leq f_{i}^{-}-i$ in the table corresponding to the upper equation will be rather complicated (in the upper equation of Example 2.4, for instance, the entries are proportional to values of the polynomial $(z-4)(z-3) z(z+6)(z+7)(z+9)$; see the proof of Theorem [7.1). However there is one easy pattern:

Corollary 2.3. Let $b_{i, j}$ be the coefficient of $\beta_{i, j}$ in the table representing the upper equation of $a$ facet $(f, \tau)$. If $j<f_{i}$, then $b_{i+1, j}=-b_{i, j}$.

The corollary says that in a table representing an upper facet equation, the diagonals from lower left to upper right have zeros in the positions below those corresponding to $f$, while above $f$ the entries are of the same absolute value but alternating sign. This behavior can be seen clearly in Example 2.4.

Proof. The lower equation has all zeros above the positions corresponding to $f^{-}$. The upper equation differs from it by a linear combination of Herzog-Kühl equations. These equations, in the case of modules of finite length that we are considering, reduce to the equations saying that the Hilbert polynomial $p_{M}$ of a module $M$ with the given Betti table is identically zero. Hilbert himself expressed $p_{M}$ in terms of the Betti table as

$$
p_{M}(d)=\sum_{j} p_{S}(d-j) \sum_{i}(-1)^{i} \beta_{i, j}(M),
$$

where $p_{S}(t)=\left(\begin{array}{c}t+n-1 \\ n-1\end{array}\right)$, regarded as a polynomial in $t$, is the Hilbert polynomial of $S$. To say that this polynomial of degree $n-1$ is identically zero is to say that it has $n$ consecutive zero values, and this is expressed by the vanishing of linear functions given by tables whose entries along the diagonals have constant absolute value and alternating signs. 
Example 2.4. The facet $((-4,-3,0,2,4,6,7,9), 3)$ involves the chain of degree sequences $f^{-}=(-4,-3,0,2,3,6,7,9), f=(-4,-3,0,2,4,6,7,9)$ and $f^{+}=$ $(-4,-3,0,3,4,6,7,9)$ indicated by the diagram

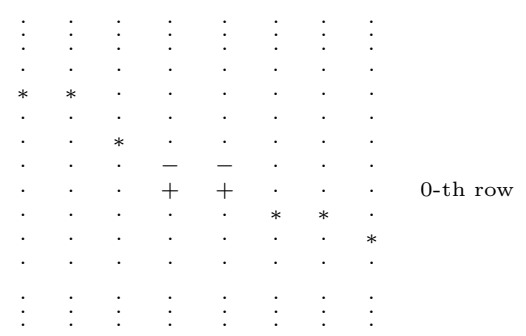

where the $\cdot$ represents a zero entry and the,-+ and $*$ are in the positions where the Betti tables corresponding to $f^{-}, f^{+}$or both are non-zero. The linear functional defining the upper hyperplane of this facet, computed by the algorithm above, is given by the dot product with the matrix

$\begin{array}{cccccccc}\vdots & \vdots & \vdots & \vdots & \vdots & \vdots & \vdots & \vdots \\ 1755 & -385 & 0 & 0 & 66 & -70 & 0 & 100 \\ 385 & 0 & 0 & -66 & 70 & 0 & -100 & 175 \\ 0^{*} & 0^{*} & 66 & -70 & 0 & 100 & -175 & 189 \\ 0 & 0 & 70 & 0 & -100 & 175 & -189 & 140 \\ 0 & 0 & 0^{*} & 100 & -175 & 189 & -140 & 60 \\ 0 & 0 & 0 & 175 & -189 & 140 & -60 & 0 \\ 0 & 0 & 0 & 0^{+} & 0^{+} & 60 & 0 & 0 \\ 0 & 0 & 0 & 0 & 0 & 0^{*} & 0^{*} & 44 \\ 0 & 0 & 0 & 0 & 0 & 0 & 0 & 0^{*} \\ 0 & 0 & 0 & 0 & 0 & 0 & 0 & 0 \\ \vdots & \vdots & \vdots & \vdots & \vdots & \vdots & \vdots & \vdots\end{array}$

The zeroes marked $0^{*}$ and $0^{+}$and all zeroes below are forced by our construction. The remaining zeroes are in diagonals containing a $0^{*}$; these are implied by Corollary 2.3 ,

\section{Linear functions non-negative on Betti tables OF FREE RESOLUTIONS}

In this section we introduce the bilinear form on Betti tables and cohomology tables that is the fundamental tool of this paper.

If $M$ is a finitely generated graded $S=K\left[x_{1}, \ldots, x_{n}\right]$-module, we set $h_{\ell}(M)=$ $\operatorname{dim}\left(M_{\ell}\right)$, the value of the Hilbert function of $M$ at $\ell$. For any bounded complex $G$ of such modules, we set $\chi(G)=\sum_{i}(-1)^{i} h_{0}\left(G_{i}\right)$.

Given a Betti table $\beta \in \mathbb{B}$ and a cohomology table $\gamma \in \mathbb{B}^{*}$ we define

$$
\langle\beta, \gamma\rangle=\sum_{\{i, j, k \mid j \leq i\}}(-1)^{i-j} \beta_{i, k} \gamma_{j,-k} .
$$

If $E$ is a bounded complex of modules with cohomological indices increasing from 0 ,

$$
E: 0 \rightarrow E^{0} \rightarrow E^{1} \rightarrow \cdots
$$

and $F$ is a free complex, then we write

$$
\langle\beta, E\rangle, \quad\langle F, \gamma\rangle, \quad \text { and } \quad\langle F, E\rangle
$$

for

$$
\langle\beta, \gamma(E)\rangle, \quad\langle\beta(F), \gamma\rangle, \quad \text { and } \quad\langle\beta(F), \gamma(E)\rangle,
$$


respectively. If $F_{i}=\sum_{k} S(-k)^{\beta_{i, k}}$, then $\sum_{k} \beta_{i, k} h_{-k}\left(H^{j}(E)\right)=h_{0}\left(F_{i} \otimes H^{j}(E)\right)$, so we can simplify the formula to

$$
\langle F, E\rangle=\sum_{j} \chi\left(F_{\geq j} \otimes H^{j}(E)\right)
$$

Thus the value of the functional $\langle-, E\rangle$ on $F$ is given by the dot product of the Betti table of $F$ with the matrix

$$
\begin{array}{cccc}
\vdots & \vdots & \vdots & \\
h_{0}^{0} & h_{1}^{1}-h_{1}^{0} & h_{2}^{2}-h_{2}^{1}+h_{2}^{0} & \ldots \\
h_{1}^{0} & h_{2}^{1}-h_{2}^{0} & h_{3}^{2}-h_{3}^{1}+h_{3}^{0} & \ldots \\
h_{2}^{0} & h_{3}^{1}-h_{3}^{0} & h_{4}^{2}-h_{4}^{1}+h_{4}^{0} & \cdots
\end{array}
$$

where $h_{j}^{i}$ denotes the dimension of the degree $j$ component of $H^{i}(E)$ and the entry $h_{0}^{0}$ is in the $(0,0)$ place.

Since these definitions involve only the cohomology of $E$, we have $\langle\beta, E\rangle=$ $\left\langle\beta, H^{*}(E)\right\rangle$, where $H^{*}(E)$ is interpreted as a complex having zero differential, and similarly for $F$-we could set the differentials of $F$ equal to zero without disturbing the definition. The importance of the complex $E$ and the differentials in $F$ appears from the following, which is the main technical result of this paper.

Theorem 3.1. If

$$
E: \quad 0 \rightarrow E^{0} \rightarrow \cdots \rightarrow E^{n} \rightarrow 0
$$

is a complex of graded free $S$-modules and $F$ is a free resolution of a graded $S$ module $M$, then

1. $\langle F, E\rangle \geq 0$.

2. Suppose in addition that $M$ and the modules $H^{j}(E)$, for $j>0$, have finite length. If

$$
\begin{aligned}
& 0>\operatorname{reg} M+\operatorname{reg} E^{0}, \text { and } \\
& 0>\operatorname{reg} F_{j-1}+\operatorname{reg} H^{j}(E) \text { for every } j>0,
\end{aligned}
$$

then $\langle F, E\rangle=0$.

Example 3.2. Suppose that

$$
\begin{gathered}
E: \quad \cdots \rightarrow 0 \rightarrow E^{j} \rightarrow 0 \rightarrow \cdots \\
j
\end{gathered}
$$

is 0 except in cohomological degree $j$ and that $F$ is the free resolution of a module $M$.

1. If $E^{j}$ is free, then

$$
\langle F, E\rangle=\chi\left(F_{\geq j} \otimes E^{j}\right)=h_{0}\left(\operatorname{Syz}_{j}(M) \otimes E^{j}\right) .
$$

It is obvious that $\langle F, E\rangle \geq 0$ in this case. 
2. If $E^{j}$ is not free, then $\langle F, E\rangle$ may be negative. For example, if $j=0$, $E=E^{j}=K$, and $F$ is the Koszul complex resolving $K(1)$, then $\langle F, E\rangle=$ $-\beta_{1,0}(K(1))=-n$. Thus we cannot drop the condition that $E$ is a free complex in Theorem 3.1 .

3. However, if $j=n, E=E^{j}=K$, and $F$ is the minimal free resolution of $M$, then $\langle F, E\rangle=\beta_{n, 0}(M) \geq 0$. This positivity could have been deduced from Theorem 3.1. since in this case we can rewrite $\langle F, E\rangle=\left\langle F, E^{\prime}\right\rangle$, where $E^{\prime}$ is a Koszul complex.

Proof of Theorem 3.1. Consider the double complex $F \otimes E$ :

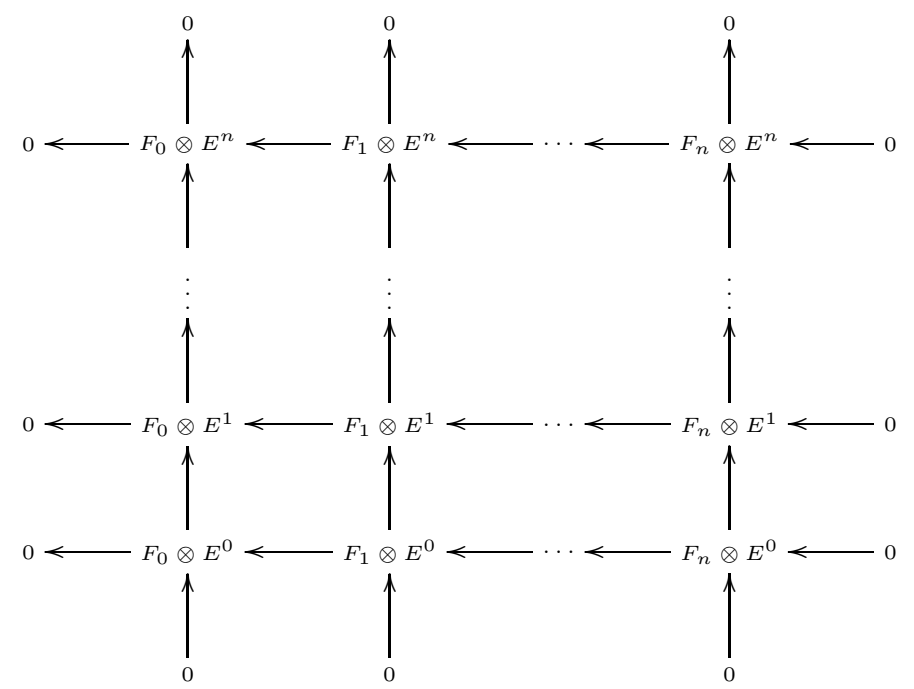

and the two spectral sequences converging to its total homology. Since $F$ is a resolution, the spectral sequence beginning with $H_{\text {vert }} H_{\text {hor }}(F \otimes E)$ degenerates at that point, and we see that the homology of the total complex $F \otimes E$ is $H^{*}(M \otimes E)$. In particular,

$$
H_{\mathrm{tot}}^{0}(F \otimes E)=\operatorname{ker}\left(M \otimes E^{0} \rightarrow M \otimes E^{1}\right)
$$

and $H_{\text {tot }}^{j}(F \otimes E)=0$ for $j<0$.

Now consider the other spectral sequence of the double complex,

$$
\mathbf{E}: \quad{ }^{1} \mathbf{E}=H_{\text {vert }}(F \otimes E) \Rightarrow H_{\text {tot }}(E \otimes F) .
$$

We work in cohomological indices, considering $F_{\ell}$ to be in cohomological degree $-\ell$. To simplify notation, set $N^{j}:=H^{j}(E)$ and write $N$ for $\sum_{j} N^{j}$.

Because each $F_{k}$ is free, $\left(H_{\text {vert }}(F \otimes E)\right)^{(j,-\ell)}=F_{\ell} \otimes N^{j}$, so the value of the functional $\langle F, E\rangle$ is the Euler characteristic of the truncation of $H_{\text {vert }}(F \otimes E)$ consisting of the terms $F_{\ell} \otimes N^{j}$ of total cohomological degree $j-\ell \leq 0$. The differential from $F$ makes this into a complex that we will call $(F \otimes N) \leq 0$, as in the following 
diagram:

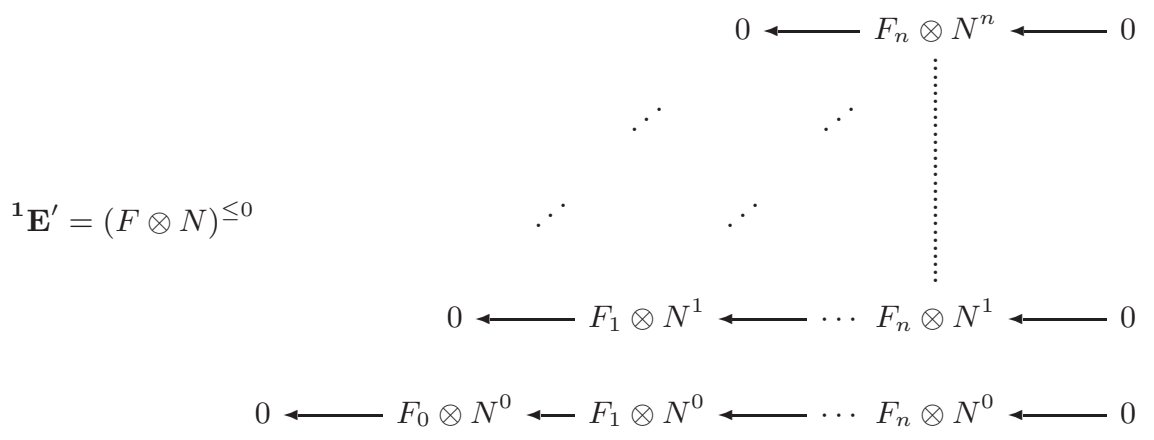

The differentials in $\mathbf{E}$ go from the diagonal of cohomological degree $-\ell-1$, represented on the first page of $\mathbf{E}$ by $\sum_{\ell} F_{i+\ell+1} \otimes N^{i}$, to the diagonal of cohomological degree $-\ell$, represented on the first page of $\mathbf{E}$ by $\sum_{\ell} F_{i+\ell} \otimes N^{i}$. Thus $\mathbf{E}$ induces a spectral sequence $\mathbf{E}^{\prime}$ whose first page is ${ }^{\mathbf{1}} \mathbf{E}^{\prime}=(F \otimes N) \leq 0$ as in the diagram above. Since taking homology preserves the Euler characteristic, we see that $\langle F, E\rangle$ is the Euler characteristic of the infinity term ${ }^{\infty} \mathbf{E}^{\prime}$.

Since ${ }^{1} \mathbf{E}^{\prime}$ is zero in strictly positive cohomological degrees, so is ${ }^{\infty} \mathbf{E}^{\prime}$. Since the total complex of $F \otimes E$ has no homology in negative cohomological degrees, the same is true of $(F \otimes E)^{\leq 0}$, so ${ }^{\infty} \mathbf{E}^{\prime}$ will also be zero in strictly negative cohomological degrees. Thus the value of the functional $\langle F, E\rangle$ is simply the dimension of the degree zero part of ${ }^{\infty} \mathbf{E}^{\prime}$. In particular, it is non-negative, proving part 1 of the theorem.

To prove the part 2 of the theorem, we compare $\mathbf{E}^{\prime}$ with $\mathbf{E}$. We write ${ }^{t} d^{\prime}$ and ${ }^{t} d$ for the differentials of the two sequences on the $t$-th page. The second differential ${ }^{2} d$ goes from the kernel of ${ }^{1} d$ to $F \otimes N$ modulo the image of ${ }^{1} d$. Thus there is a well-defined submodule of $F \otimes N$ that we may denote by image $\left({ }^{1} d\right)+$ image $\left({ }^{2} d\right)$, even though image $\left({ }^{2} d\right)$ is not itself a submodule of $F \otimes N$. Similarly, it makes sense to speak of the submodules

$$
\sum_{t \geq 1} \text { image }{ }^{t} d \subset \bigcap_{t \geq 1} \operatorname{ker}^{t} d \subset F \otimes N .
$$

To simplify the notation in this and the next proof set

$$
\begin{aligned}
K_{t}^{i} & =\left(\sum_{\ell} F_{\ell} \otimes N^{\ell+i}\right) \cap \bigcap_{s \leq t} \operatorname{ker}^{s} d, \\
I_{t}^{i} & =\left(\sum_{\ell} F_{\ell} \otimes N^{\ell+i}\right) \cap \sum_{s \leq t} \operatorname{image}^{s} d,
\end{aligned}
$$

for $1 \leq t \leq \infty$. Further, let $K_{0}^{i}=\sum_{\ell} F_{\ell} \otimes N^{\ell+i}$ and $I_{0}^{i}=0$.

The spectral sequence $\mathbf{E}$ gives a filtration of $H_{\text {tot }}(F \otimes E)$ whose associated graded module is ${ }^{\infty} \mathbf{E}=K_{0}^{\infty} / I_{0}^{\infty}$. Since the differentials ${ }^{t} d$ coming into the cohomological degree 0 diagonal terms of $\mathbf{E}$ coincide with the ${ }^{t} d^{\prime}$, we have ${ }^{\infty} \mathbf{E}^{\prime}=$ $\left(\sum_{\ell} F_{\ell} \otimes N^{\ell}\right) / I_{\infty}^{0}$. Thus

$$
\langle F, E\rangle=h_{0}\left({ }^{\infty} \mathbf{E}^{\prime}\right)=h_{0}\left(H_{\text {tot }}^{0}(F \otimes E)\right)+h_{0}\left(\frac{\sum_{\ell} F_{\ell} \otimes N^{\ell}}{K_{\infty}}\right) .
$$


The module $\left(\sum_{\ell} F_{\ell} \otimes N^{\ell}\right) / K_{\infty}$ is filtered by the submodules $K_{t} / K_{\infty}$. The module $\sum_{\ell} F_{\ell} \otimes N^{\ell+1}$ is filtered by the $I_{t}^{1}$, and the differential ${ }^{t+1} d$ identifies the factor $K_{t}^{0} / K_{t+1}^{0}$ with $I_{t+1}^{1} / I_{t}^{1}$. As already noted, we have $H_{\text {tot }}^{0}(F \otimes E) \subset M \otimes E^{0}$.

Together with part 1 of the theorem, these observations yield

$$
0 \leq\langle E, F\rangle \leq h_{0}\left(M \otimes E^{0}\right)+h_{0}\left(\sum_{\ell} F_{\ell} \otimes N^{\ell+1}\right) .
$$

Under the hypotheses of of part 2 of the theorem, both $M \otimes E^{0}$ and $\sum_{\ell} F_{\ell} \otimes N^{\ell+1}$ are zero in degree 0 , proving the vanishing of $\langle E, F\rangle$ in this case.

\section{Linear FUnCtions NON-NEGATIVE \\ on BetTi tables of Minimal FREE RESOlutions}

In this section we construct a family of bilinear functions on Betti tables and cohomology tables that are modifications of $\langle\beta, \gamma\rangle$. When $\gamma=\gamma(E)$ for a free complex $E$ and $\beta=\beta(F)$ for a minimal free resolution, the result is non-negative, but it can be negative when $F$ is non-minimal. Since the construction is somewhat opaque, we begin by explaining it from several points of view.

Recall from $\$ 3$ that

$$
\langle\beta, \gamma\rangle=\sum_{\{i, j, k \mid j \leq i\}}(-1)^{i-j} \beta_{i, k} \gamma_{j,-k} .
$$

Given a cohomological index $\tau$ and a degree bound $c$ we set

$$
\begin{aligned}
& \langle\beta, \gamma\rangle_{c, \tau}=\sum \quad(-1)^{i-j} \beta_{i, k} \gamma_{j,-k} \\
& +\sum_{\{i, j, k, \epsilon \mid 0 \leq \epsilon \leq 1, \quad j=\tau, \quad}^{\{i, j, k \mid j \leq i}{ }_{i=j+\epsilon, \quad k \leq c+\epsilon\}}^{\text {and }(j<\tau}(-1)^{i-j} \beta_{i, k} \gamma_{j,-k} .
\end{aligned}
$$

It may help the reader to see the coefficient of each $\beta_{i, i+\ell}$ (the entry in column $i$, row $\ell$ ) in the functional $\langle\beta, \gamma,\rangle_{\tau, c}$ explicitly. The formula depends on which region of the Betti table the index $(i, \ell)$ falls into, as follows:

\begin{tabular}{c|ccc} 
& $i<\tau$ & $\tau \leq i \leq \tau+1$ & $\tau+1<i$ \\
\hline$\ell \leq c-\tau$ & $U$ & $V^{\prime}$ & $W$ \\
$\ell>c-\tau$ & $U$ & $V$ & $W$
\end{tabular}

Recall that in the row labelled $\ell$ and the column labelled $i$ the entry of the Betti table is $\beta_{i, i+\ell}$. The coefficient of $\beta_{i, i+\ell}$ in the unmodified functional $\langle\beta, \gamma$,$\rangle is$ $\sum_{j \leq i}(-1)^{i-j} \gamma_{j,-i-\ell}$. This differs from the coefficient in the modified functional only in the range of $j$ in the summation. Region by region, as in the diagram above, the coefficient of $\beta_{i, i+\ell}$ is:

$U: \sum_{\{j \mid j \leq i\}}(-1)^{i-j} \gamma_{j,-i-\ell}$ (as for the unmodified functional $\langle\beta, \gamma$,$\rangle ),$

$V: \sum_{\{j \mid j \leq \tau-1\}}(-1)^{i-j} \gamma_{j,-i-\ell}$

$V^{\prime}: \sum_{\{j \mid j \leq \tau\}}(-1)^{i-j} \gamma_{j,-i-\ell}$,

$W: \sum_{\{j \mid j \leq i-2\}}(-1)^{i-j} \gamma_{j,-i-\ell}$.

We were originally led to consider the functionals $\langle\beta(F), \gamma(E)\rangle_{c, \tau}$ by the inspection of the upper facet equations in many examples. The reader may also understand the idea better from Examples 4.3 and 8.3 . 
We can follow the modification of the functional through the proof of Theorem 3.1 as well. The formulas say that, applied to a free resolution $F$, the value $\langle F, E\rangle_{c, \tau}:=$ $\langle\beta(F), \gamma(E)\rangle_{c, \tau}$ is constructed from $\langle F, E\rangle$ by changing the upper parts of the two leftmost diagonals in the complex ${ }^{\mathbf{1}} \mathbf{E}^{\prime}$ in the proof of Theorem 3.1. The following diagram shows the location of the changes we make to obtain the new function when $n=7, \tau=3$, the case of Example 2.4

$\begin{array}{lllllllll}0 & 0 & 0 & 0 & 0 & 0 & 0 & Z & \\ 0 & 0 & 0 & 0 & 0 & 0 & Z & Z & \\ 0 & 0 & 0 & 0 & 0 & Z & Z & * & \\ 0 & 0 & 0 & 0 & Z & Z & * & * & \\ 0 & 0 & 0 & C & C & * & * & * & \tau \text {-th row } \\ 0 & 0 & * & * & * & * & * & * & \\ 0 & * & * & * & * & * & * & * & \\ * & * & * & * & * & * & * & * & \end{array}$

The entries marked $*$ in this diagram correspond to terms that are equal to $F_{i} \otimes N^{j}$ as before, the entries $Z$ are set equal to zero, and the entries $C$ are altered by a degree restriction. The more general construction given in the proof of Theorem 4.1 provides a more conceptual picture of what is going on.

Theorem 4.1. If $\beta$ is the Betti diagram of a minimal free resolution of a graded $S$-module, then $\langle\beta, E\rangle_{c, \tau} \geq 0$.

Proof. To clarify the proof we formulate a more general result. Suppose we are in the situation of part 1 of Theorem 3.1 and that we are given submodules $A_{\ell}^{\prime} \subset$ $F_{\ell} \otimes N^{\ell}$ and $B_{\ell}^{\prime} \subset F_{\ell+1} \otimes N^{\ell}$ for each $\ell$. Set

$$
\begin{aligned}
A^{\prime}:=\sum_{\ell} A_{\ell}^{\prime} \subset \quad A:=\sum_{\ell} F_{\ell} \otimes N^{\ell}, \\
B^{\prime}:=\sum_{\ell} B_{\ell}^{\prime} \subset \quad B:=\sum_{\ell} F_{\ell+1} \otimes N^{\ell} .
\end{aligned}
$$

Let $D$ denote the diagram of modules that agrees with ${ }^{1} \mathbf{E}^{\prime}=(F \otimes N) \leq 0$ except for the first two diagonals, where the terms $F_{\ell} \otimes N^{\ell}$ and $F_{\ell+1} \otimes N^{\ell}$ have been replaced by the terms $A_{\ell}^{\prime}$ and $B_{\ell}^{\prime}$ respectively, so that $A$ and $B$ are replaced by $A^{\prime}$ and $B^{\prime}$ (and we forget, for a moment, the maps). Set

$$
\chi(D):=\sum_{j \leq i}(-1)^{i-j} D_{i}^{j}=\langle F, E\rangle-h_{0} A+h_{0} A^{\prime}+h_{0} B-h_{0} B^{\prime} .
$$

Let ${ }^{t} d$ be the differentials of the spectral sequence $\mathbf{E}$. With notation as in the proof of Theorem 3.1, the spectral sequence gives us sequences of submodules $K_{t}^{-1} \subset B$ and $I_{t}^{0} \subset A$, such that ${ }^{t+1} d$ identifies $K_{t}^{-1} / K_{t+1}^{-1}$ with $I_{t+1}^{0} / I_{t}^{0}$.

Lemma 4.2. If

$$
{ }^{t+1} d\left(B^{\prime} \cap K_{t}^{-1}\right) \subset\left(A^{\prime}+I_{t}^{0}\right) / I_{t}^{0}
$$

for all $t \geq 0$, then $\chi(D) \geq 0$.

Proof of Lemma 4.2. Set

$$
\begin{aligned}
\bar{B} & =K_{\infty}^{-1} \oplus \bigoplus_{t \geq 0} \frac{K_{t}^{-1}}{K_{t+1}^{-1}} \\
\bar{A} & =\frac{\sum_{\ell} F_{\ell} \otimes N^{\ell}}{I_{\infty}^{0}} \oplus \bigoplus_{t \geq 0} \frac{I_{t+1}^{0}}{I_{t}^{0}} .
\end{aligned}
$$


Let $\alpha: \bar{B} \rightarrow \bar{A}$ be the map taking $K_{\infty}^{-1}$ to zero and identifying $K_{t}^{-1} / K_{t+1}^{-1}$ with $I_{t+1}^{0} / I_{t}^{0}$ by means of ${ }^{t+1} d$.

Our hypothesis shows that $\alpha$ induces a map of associated graded modules $\overline{B^{\prime}} \rightarrow$ $\overline{A^{\prime}}$ coming from the induced filtrations. Thus we have a commutative diagram with exact rows and columns:

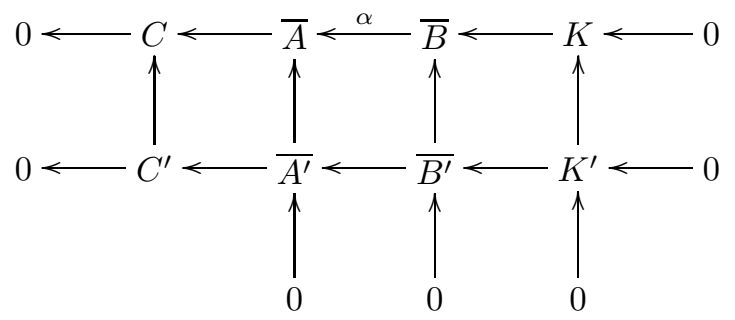

where $C=\left(\sum_{\ell} F_{\ell} \otimes N^{\ell}\right) / I_{\infty}^{0}, K=K_{\infty}^{-1}$, and $K^{\prime}, C^{\prime}$ are the kernel and cokernel of the map induced by $\alpha$. The proof of Theorem 3.1 shows that $\langle F, E\rangle=h_{0} C$, so $\chi D=h_{0} C-h_{0} \bar{A}+h_{0} \overline{A^{\prime}}+h_{0} \bar{B}-h_{0} \overline{B^{\prime}}$, and an easy diagram chase shows that this is equal to $\left(h_{0} K-h_{0} K^{\prime}\right)+h_{0} C^{\prime}$. Since $K^{\prime} \subset K$, this is non-negative, as required.

Proof of Theorem 4.1 continued. To obtain $\langle F, E\rangle_{c, \tau}$ we consider

$$
F_{i}^{a}= \begin{cases}F_{i} & \text { if } i<\tau, \\ \sum_{j \leq c} S(-j)^{\beta_{\tau, j}} & \text { if } i=\tau, \\ 0 & \text { if } i>\tau\end{cases}
$$

and

$$
F_{i}^{b}= \begin{cases}F_{i} & \text { if } i<\tau+1 \\ \sum_{j \leq c+1} S(-j)^{\beta_{\tau+1, j}} & \text { if } i=\tau+1 \\ 0 & \text { if } i>\tau+1\end{cases}
$$

Let

$$
A_{\ell}^{\prime}=F_{\ell}^{a} \otimes N^{\ell} \subset F_{\ell} \otimes N^{\ell}
$$

and

$$
B_{\ell}^{\prime}=F_{\ell+1}^{b} \otimes N^{\ell} \subset F_{\ell+1} \otimes N^{\ell} .
$$

Since $F$ is minimal, the differential of $F$ maps $F_{\tau+1}^{b}$ to $F_{\tau}^{a}$, and it follows that $A^{\prime}, B^{\prime}$ satisfy the hypotheses of Lemma 4.2 for ${ }^{1} d$. For $t>1$ the hypotheses are trivially satisfied: the map

$$
{ }^{t+1} d: B_{\ell}^{\prime} \cap K_{t}^{-1} \rightarrow F_{\ell-t+1} /\left(I_{t}^{0} \cap F_{\ell-t+1}\right)
$$

has source $=0$ for $\ell>\tau$, while for $\ell \leq \tau, F_{\ell-t+1}=F_{\ell-t+1}^{a}$, so $A=A^{\prime}$ in this component. Moreover the resulting diagram $D$ satisfies $\chi D=\langle F, E\rangle_{c, \tau}$. By Lemma 4.2 we have $\langle F, E\rangle_{c, \tau} \geq 0$. 
Example 4.3. The upper and lower equation of the facet facet $((-1,0,2,3), 1)$ have coefficients as indicated in the following tables:

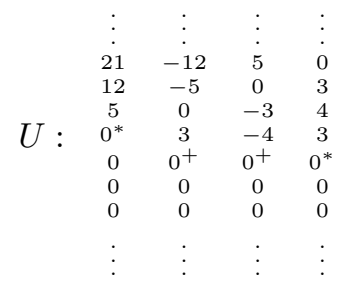

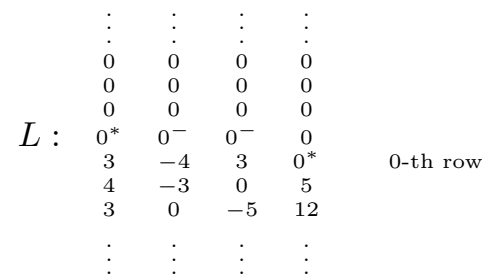

If $M$ is any graded module with this Betti table and $p_{M}$ is its Hilbert polynomial, then one computes $U(\beta(M))-L(\beta(M))=5 p_{M}(1)-3 p_{M}(2)$. Thus we are led to consider a complex

$$
E: \quad 0 \rightarrow E^{0}=S(1)^{5} \rightarrow E^{1}=S(2)^{3} \rightarrow 0 .
$$

The equation $\langle-, E\rangle$ obtained from this complex has coefficients

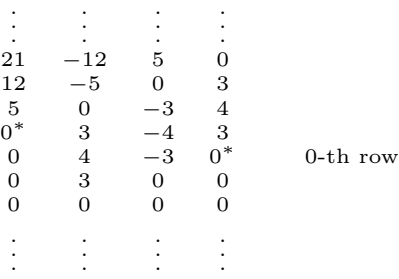

To obtain the desired facet equation, we take $\tau=1, c=0$. The functional $\langle-, E\rangle_{0,1}$ is the result of replacing the lowest 4 and the lowest two $3 \mathrm{~s}$ by zero, obtaining the table $U$.

Notice that $\langle F, E\rangle_{c, \tau}$ is negative for some non-minimal resolutions $F$. For example, if we add to the minimal resolution of $M=K(1)$ and a trival complex as follows:

$$
\begin{aligned}
& 0 \leftarrow S(1) \leftarrow S^{3} \leftarrow S(-1)^{3} \leftarrow S(-2) \leftarrow 0 \\
& +0 \leftarrow 0 \leftarrow S(-1) \leftarrow S(-1) \leftarrow 0 \leftarrow 0
\end{aligned}
$$

we get a non-minimal resolution $F$ with Betti table

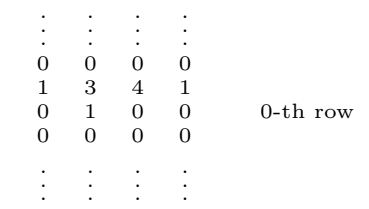

and we compute $\langle F, E\rangle_{0,1}=-4<0$.

\section{The EXISTENCE OF PURE RESOLUTIONS}

Theorem 5.1. Let $K$ be any field, and let $d=\left(d_{0}<\cdots<d_{n}\right)$ be a sequence of integers. There exists a graded $K\left[x_{1}, \ldots, x_{n}\right]$-module of finite length with $\beta_{0}=$ $\prod_{i=1}^{n}\left(\begin{array}{c}d_{i}-d_{0}-1 \\ d_{i}-d_{i-1}-1\end{array}\right)$ generators, whose minmal free resolution is pure with degree sequence $d$. 
In the case where $d_{i}-d_{i-1}=1$ for all but one value of $i$, the complexes we produce coincide with those of Buchsbaum-Eisenbud [1973a] and Kirby [1974] (see Eisenbud [1995], Appendix A 2.6 for an exposition).

Our construction (and that for supernatural sheaves given in $\sqrt[6]{6}$ ) starts from a sequence of multilinear forms on a product of projective spaces posessing a property that we could deduce from a general position argument if the ground field were assumed infinite. Since we do not wish to make that assumption, we give a direct construction.

Proposition 5.2. Let $m_{0}, \ldots, m_{k}$ be non-negative integers, and let the homogeneous coordinates on $\mathbb{P}^{m_{j}}$ be $x_{0}^{(j)}, \ldots, x_{m_{j}}^{(j)}$. The multilinear forms

$$
x_{\ell}=\sum_{\mu_{0}+\cdots+\mu_{k}=\ell} \prod_{j=0}^{k} x_{\mu_{j}}^{(j)} \quad \text { for } \ell=0, \ldots, \sum_{j=0}^{k} m_{j}
$$

have no common zeros in

$$
\mathbb{P}_{K}^{m_{0}} \times \mathbb{P}_{K}^{m_{1}} \times \cdots \times \mathbb{P}_{K}^{m_{k}}
$$

Proof. We do induction on $M:=\sum_{j=0}^{k} m_{j}$. The case $M=0$ is trivial. Suppose that $M>0$ and the $x_{\ell}$ all vanish at a point $P \in \prod \mathbb{P}^{m_{j}}$. In particular,

$$
x_{M}=\prod_{j=0}^{k} x_{m_{j}}^{(j)}(P)=0 .
$$

We cannot have $x_{m_{j}}^{(j)}(P)=0$ for $j$ such that $m_{j}=0$, so $x_{m_{j}}^{(j)}(P)=0$ for some $j$ with $m_{j} \geq 1$. Write $\mathbb{P}^{m_{j}-1}$ for the subspace of $\mathbb{P}^{m_{j}}$ where $x_{m_{j}}^{(j)}$ vanishes. The forms $x_{0}, \ldots, x_{M-1}$ restrict to the corresponding set of forms on

$$
\mathbb{P}^{m_{0}} \times \cdots \times \mathbb{P}^{m_{j}-1} \times \cdots \times \mathbb{P}^{m_{k}}
$$

and vanish at $P$ there, contradicting the inductive hypothesis.

We will construct pure resolutions by pushing forward other pure resolutions, starting with the Koszul complex. The following result is the key to this process. For any product $X_{1} \times X_{2}$ with projections $p: X_{1} \times X_{2} \rightarrow X_{1}$ and $q: X_{1} \times X_{2} \rightarrow X_{2}$ and sheaves $\mathcal{L}_{i}$ on $X_{i}$, we set

$$
\mathcal{L}_{1} \otimes \mathcal{L}_{2}:=p^{*} \mathcal{L}_{1} \otimes q^{*} \mathcal{L}_{2}
$$

Proposition 5.3. Let $\mathcal{F}$ be a sheaf on $X \times \mathbb{P}^{m}$, and let $p: X \times \mathbb{P}^{m} \rightarrow X$ be the projection. Suppose that $\mathcal{F}$ has a resolution of the form

$$
\mathcal{G}: 0 \rightarrow \mathcal{G}_{N} \otimes \mathcal{O}\left(-e_{N}\right) \rightarrow \cdots \rightarrow \mathcal{G}_{0} \otimes \mathcal{O}\left(-e_{0}\right) \rightarrow \mathcal{F} \rightarrow 0
$$

with degrees $e_{0}<\cdots<e_{N}$. If this sequence contains the subsequence $\left(e_{k+1}, \ldots, e_{k+m}\right)$ $=(1,2, \ldots, m)$ for some $k \geq-1$, then

$$
R^{\ell} p_{*} \mathcal{F}=0 \text { for } \ell>0
$$

and $p_{*} \mathcal{F}$ has a resolution on $X$ of the form

$$
\begin{aligned}
0 \rightarrow \mathcal{G}_{N} \otimes H^{m} \mathcal{O}\left(-e_{N}\right) \rightarrow \cdots & \rightarrow \mathcal{G}_{k+m+1} \otimes H^{m} \mathcal{O}\left(-e_{k+m+1}\right) \stackrel{\phi}{\rightarrow} \mathcal{G}_{k} \otimes H^{0} \mathcal{O}\left(-e_{k}\right) \rightarrow \cdots \\
\cdots & \cdots \rightarrow \mathcal{G}_{0} \otimes H^{0} \mathcal{O}\left(-e_{0}\right) .
\end{aligned}
$$


Proof. We may suppose $m>0$. From the numerical hypotheses we see that $e_{i} \leq 0$ for $i \leq k$ and $e_{i} \geq m+1$ for $i \geq k+m+1$.

Consider the spectral sequence

$$
E_{1}^{i,-j}=R^{i} p_{*}\left(\mathcal{G}_{j} \otimes \mathcal{O}\left(-e_{j}\right)\right) \Rightarrow R^{i-j} p_{*} \mathcal{F} .
$$

By the projection formula, the terms of the $E_{1}$ page are

$$
R^{i} p_{*}\left(\mathcal{G}_{j} \otimes \mathcal{O}\left(-e_{j}\right)\right)= \begin{cases}\mathcal{G}_{j} \otimes H^{m}\left(\mathbb{P}^{m}, \mathcal{O}\left(-e_{j}\right)\right) & \text { if } j \geq k+m+1 \text { and } i=m, \\ \mathcal{G}_{j} \otimes H^{0}\left(\mathbb{P}^{m}, \mathcal{O}\left(-e_{j}\right)\right) & \text { if } j \leq k \text { and } i=0, \\ 0 & \text { otherwise. }\end{cases}
$$

Thus the spectral sequence degenerates to the complex (1), where $\phi$ is a differential from the $m$-th page and the other maps are differentials from the first page. In particular only terms $E_{\infty}^{i,-j}$ with $i \leq j$ can be non-zero. On the other hand, the terms $R^{i-j} p_{*} \mathcal{F}$ can be non-zero only for $i \geq j$. Hence the complex (1) is exact and resolves $\bigoplus_{i \geq 0} E_{\infty}^{i,-i}=E_{\infty}^{0,0}=p_{*} \mathcal{F}$, while the higher direct images of $\mathcal{F}$ vanish.

Proof of Theorem 5.1. To simplify the notation we may harmlessly assume that $d_{0}=0$. Let $m_{0}=m=n-1$, and for $i=1, \ldots, n$ set $m_{i}=d_{i}-d_{i-1}-1$ and set $M=\sum_{j=0}^{k} m_{j}=d_{n}-1$. Choose $M+1$ homogenous forms of multidegree $(1, \ldots, 1)$ without a common zero on

$$
\mathbb{P}:=\mathbb{P}^{m} \times \mathbb{P}^{m_{1}} \times \cdots \times \mathbb{P}^{m_{n}}
$$

such as the forms described in Proposition [5.2. Let

$$
\mathcal{K}: 0 \rightarrow \mathcal{K}_{M+1} \rightarrow \cdots \rightarrow \mathcal{K}_{0} \rightarrow 0
$$

be the tensor product of the Koszul complex of these forms on $\mathbb{P}$ and the line bundle $\mathcal{O}_{\mathbb{P}}\left(0,0, d_{1}, \ldots, d_{n-1}\right)$, so $\mathcal{K}_{i}=\mathcal{O}_{\mathbb{P}}\left(-i,-i, \ldots, d_{n-1}-i\right)^{\left(\begin{array}{c}d_{n} \\ i\end{array}\right)}$ for $i=0, \ldots, d_{n}$. Let

$$
\pi: \mathbb{P}^{m} \times \mathbb{P}^{m_{1}} \times \cdots \times \mathbb{P}^{m_{n}} \rightarrow \mathbb{P}^{m}
$$

be the projection onto the first factor. The complex $\mathcal{K}$ is exact because the forms have no common zero. Hence $\mathbf{R} \pi_{*}(\mathcal{K})=0$.

The choices of these particular twists can be understood from the following table, which gives some of the important ones, starting from the left hand end of $\mathcal{K}$ :

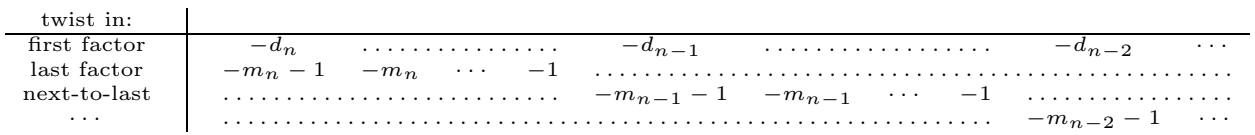

If we think of $\mathcal{K}$ as a resolution of the zero sheaf $\mathcal{F}=0$ and factor $\pi$ into the successive projections along the factors of the product $\mathbb{P}^{m_{1}} \times \cdots \times \mathbb{P}^{m_{n}}$, then we may use Proposition 5.3 repeatedly to get a resolution of $\pi_{*} \mathcal{F}=0$ that has the form

$$
0 \rightarrow \mathcal{O}^{\beta_{n}}\left(-d_{n}\right) \rightarrow \cdots \rightarrow \mathcal{O}^{\beta_{1}}\left(-d_{1}\right) \rightarrow \mathcal{O}^{\beta_{0}} .
$$

Taking global sections in all twists, we get a complex

$$
0 \rightarrow S^{\beta_{n}}\left(-d_{n}\right) \rightarrow \cdots \rightarrow S^{\beta_{1}}\left(-d_{1}\right) \rightarrow S^{\beta_{0}}
$$

that has homology of finite length. Since the length of this complex is only $n$, the Lemme d'Acyclicité of Peskine and Szpiro [1973] (see also Buchsbaum and Eisenbud [1973b]) shows that the complex is actually acyclic. Thus it is a pure minimal resolution, with the desired degree sequence, of a graded module of finite length. 
Following the projections step by step, we see that

$$
\beta_{0}=\prod_{i=1}^{n} h^{0}\left(\mathbb{P}^{m_{i}}, \mathcal{O}_{\mathbb{P}^{m_{i}}}\left(d_{i-1}\right)\right)=\prod_{i=1}^{n}\left(\begin{array}{c}
d_{i}-1 \\
d_{i}-d_{i-1}-1
\end{array}\right) .
$$

Taking into account that a shift of all the $d_{i}$ by $d_{0}$ does not affect $\beta_{0}$, we get the formula for $\beta_{0}$ in the theorem.

\section{Sheaves With SUPERnAtural COHOMOlOGY}

In this section we work with coherent sheaves on a projective space $\mathbb{P}^{m}$. We begin by proving the existence of supernatural vector bundles, Theorem 0.4 , in a slightly sharper form.

Theorem 6.1. Let $K$ be any field. Suppose that $m=\sum_{j=1}^{k} m_{j}$ with $m_{j}>0$. If the sequence of integers $z=\left(z_{1}>\cdots>z_{m}\right)$ consists of $k$ disjoint subsequences of consecutive integers, of lengths $m_{1}, \ldots, m_{k}$, then there exists a supernatural vector bundle $\mathcal{E}$ on $\mathbb{P}^{m}$ that is defined over $K$, has root sequence $z$, and has rank $\left(\begin{array}{c}m \\ m_{1}, \ldots, m_{k}\end{array}\right)$.

Here $\left(\begin{array}{c}m \\ m_{1}, \ldots, m_{k}\end{array}\right)$ denotes the multinomial coefficient $\frac{m !}{\prod_{1}^{k} m_{j} !}$.

Proof. Let $\nu_{j}$ denote the starting index of the $j$-th subsequence, so that

$$
z_{\nu_{j}}, \ldots, z_{\nu_{j}+m_{j}-1}
$$

are consecutive. Consider the product

$$
\mathbb{P}^{m_{1}} \times \cdots \times \mathbb{P}^{m_{k}}
$$

of $k$ projective spaces and the line bundle

$$
\mathcal{L}=\mathcal{O}\left(-z_{\nu_{1}}-1, \ldots,-z_{\nu_{k}}-1\right):=p_{1}^{*} \mathcal{O}\left(-z_{\nu_{1}}-1\right) \otimes \cdots \otimes p_{k}^{*} \mathcal{O}\left(-z_{\nu_{k}}-1\right)
$$

on it.

Let

$$
\pi: \mathbb{P}^{m_{1}} \times \cdots \times \mathbb{P}^{m_{k}} \rightarrow \mathbb{P}^{m}
$$

be a finite morphism of degree defined by linear projection from the Segre embedding of the product. For an explicit example showing that this can be defined over any field $K$, we can take $\pi$ to be the map defined by the $m+1$ multilinear forms

$$
x_{\ell}=\sum_{\mu_{1}+\cdots+\mu_{k}=\ell} \prod_{j=1}^{k} x_{\mu_{j}}^{(j)} \quad \text { for } \ell=0, \ldots, m,
$$

as in Proposition 5.2 .

The desired supernatural bundle is $\mathcal{E}=\pi_{*} \mathcal{L}$. Indeed, since $\pi$ is a finite morphism we have

$$
H^{i} \mathcal{E}(d) \cong H^{i}\left(\mathbb{P}^{m_{1}} \times \cdots \times \mathbb{P}^{m_{k}}, \mathcal{L}(d, \ldots, d)\right),
$$

which we can calculate from the the Künneth formula. We see that $\mathcal{E}$ has supernatural cohomology, and its rank is

$$
\operatorname{rank} \mathcal{E}=\operatorname{deg} \pi=\left(\begin{array}{c}
m \\
m_{1}, \ldots, m_{k}
\end{array}\right) .
$$


Here is an alternate, characteristic zero construction of supernatural bundles, using representation theory. It is restricted to characteristic zero by the use of Bott's Vanishing Theorem, but we include it because it yields bundles whose rank is often not an integral multiple of the ranks of the bundles produced in Theorem 6.1 .

Theorem 6.2. Let $K$ be a field of characteristic zero. If $z=\left(z_{1}>\cdots>z_{m}\right)$ is a sequence of integers, then there exists a $G L_{m+1}$-equivariant vector bundle $\mathcal{E}$ on $\mathbb{P}_{K}^{m}$ with supernatural cohomology and root sequence $z$.

Proof. The desired vector bundles, up to a twist, can be constructed by applying Schur functors to the tautological rank $m$ quotient bundle $Q$ on $\mathbb{P}^{m}$ : Bott's Theorem (see for example Weyman [2003]) says that if $1 \leq i \leq m-1$, then the cohomology group $H^{i}\left(\left(S_{\lambda} Q\right)(d)\right)$ is non-zero if and only if $\lambda_{m-i+1}<-d-i \leq \lambda_{m-i}$, while $H^{0} S_{\lambda} Q(d)=0$ if and only if $d<0$ and $H^{m} S_{\lambda} Q(d)=0$ if and only if $m \geq$ $-m-\lambda_{1}-1$.

To obtain a desired bundle with supernatural cohomology as in the theorem we choose

$$
\lambda_{i}=z_{1}-z_{m+1-i}-m+i \quad \text { for } \quad 1 \leq i \leq m-1,
$$

and take $\mathcal{E}=\left(S_{\lambda} Q\right)\left(-z_{1}-1\right)$.

This result has an appealingly transparent statement in terms of Betti diagrams of Tate resolutions. For a partition $\lambda=\left(\lambda_{1} \geq \lambda_{2} \geq \cdots \geq \lambda_{m-1}\right)$, say $\lambda=(8,7,7,2,0)=(8,7,7,2)$, which corresponds to Example 2.4. the Tate resolution of the homogeneous bundle $S_{\lambda} Q$ has non-zero terms only in the degrees marked $*$ in the following Betti diagram, in which the Ferrers diagram is indicated:

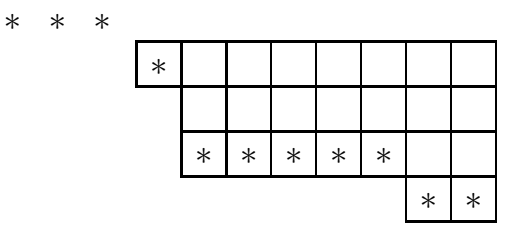

See Theorem 5.6 in Eisenbud-Schreyer 2003 .

We want to characterize the cohomology tables of supernatural bundles, and to this end we next record a basic fact about cohomology tables:

Proposition 6.3. If $\mathcal{E}$ is a non-zero coherent sheaf on $\mathbb{P}^{m}$, then every column of the cohomology table of $\mathcal{E}$ contains a non-zero entry; that is, for each integer $d$ some $\gamma_{i, i-d}(\mathcal{E}):=h^{i} \mathcal{E}(d-i) \neq 0$. Furthermore,

$$
\begin{aligned}
d \mapsto M_{d} & :=\max \left\{i \mid \gamma_{i, d-i} \neq 0\right\} \quad \text { and } \\
d \mapsto m_{d} & :=\min \left\{i \mid \gamma_{i, d-i} \neq 0\right\}
\end{aligned}
$$

are weakly decreasing functions of $d$.

Proof. The Tate resolution $\mathbb{T}(\mathcal{E})$ is a minimal free doubly infinite exact complex over the exterior algebra $\Lambda$ on $n$ generators of degree -1 . By Eisenbud-FløystadSchreyer [2003], Theorem 4.1, the term of $\mathbb{T}(\mathcal{E})$ having cohomological degree $d$ is

$$
\bigoplus_{j} H^{j}(\mathcal{E}(d-j)) \otimes \Lambda(j-d) \text {. }
$$


Moreover, since $\Lambda$ is self-injective, the dual of $\mathbb{T}(\mathcal{E})$ is also a resolution. Therefore, no term of $\mathbb{T}(\mathcal{E})$ can be zero, which gives the first statement. Since the generators of the exterior algebra are of negative degree, there are non-zero maps $\Lambda(-d) \rightarrow \Lambda(-e)$ only if $d \leq e$. Since $\mathbb{T}(\mathcal{E})$ is a minimal complex, it cannot have any maps of degree 0 . Hence $M_{d}$ is weakly decreasing. For $m_{d}$ we apply the same argument to the dual of $\mathbb{T}(\mathcal{E})$ and obtain that $-m_{-d}$ is a weakly decreasing function. Hence $m_{d}$ is a weakly decreasing function as well.

By the rank of a coherent sheaf $\mathcal{E}$ on $\mathbb{P}^{m}$ we mean the normalized leading coefficient of the Hilbert polynomial,

$$
\chi(\mathcal{E}(d))=\frac{\operatorname{rank} \mathcal{E}}{s !} d^{s}+O\left(d^{s-1}\right),
$$

where $s=\operatorname{dim} \mathcal{E}$.

Theorem 6.4. If $\mathcal{E}$ is a supernatural sheaf of dimension $s$ with root sequence $z_{1}>\cdots>z_{s}$, and if we set $z_{0}=\infty$ and $z_{s+1}=-\infty$, then, for each $0 \leq j \leq s$,

$$
h^{j} \mathcal{E}(d)= \begin{cases}\frac{\operatorname{rank} \mathcal{E}}{s !} \prod_{i=1}^{s}\left|d-z_{i}\right| & \text { if } z_{j}>d>z_{j+1}, \\ 0 & \text { otherwise. }\end{cases}
$$

Proof. Define $M_{d}, m_{d}$ as in Proposition 6.3. Of course $M_{d} \geq m_{d} \geq 0$, and by Serre's Vanishing Theorem we have $M_{d}=m_{d}=0$ for $d \gg 0$. Since the Hilbert polynomial of $\mathcal{E}$ has $s$ zeros, the dimension of the support of $\mathcal{E}$ is $s$. It follows that $H^{j} \mathcal{E}(d)=0$ for $j>s$ and any $d$, while if $d \ll 0$, then $H^{s} \mathcal{E}(d) \neq 0$. Thus $M_{d}=s$ for $d \ll 0$. Since $\mathcal{E}$ has natural cohomology this implies $m_{d}=s$ for $d \ll 0$ as well. By Proposition 6.3, $M_{d}$ and $m_{d}$ are weakly decreasing from $s$ to 0 .

Since the $M_{d}$ are weakly decreasing, the sequence of numbers $d-M_{d}$ is strictly increasing. It omits precisely those values $z$ such that $z=d-i$ with $M_{d}>i \geq M_{d+1}$. This means that precisely $s$ distinct values are omitted from the sequence $d-M_{d}$. Exactly the same considerations apply to the sequence $d-m_{d}$.

If $\mathcal{E}$ has natural cohomology, then the vanishing of $\chi(\mathcal{E}(z))$ implies the vanishing of all $H^{j}(\mathcal{E}(z))$, so the integral roots of the Hilbert polynomial must be among the omitted values of the sequences $\left\{d-M_{d}\right\}$ and $\left\{d-m_{d}\right\}$. If $\mathcal{E}$ has supernatural cohomology, then there are $s$ integral roots, which thus give all the omitted values. It follows that the omitted values are the same for $\left\{d-M_{d}\right\}$ and $\left\{d-m_{d}\right\}$. Since these two sequences are the same for $d \ll 0$, they must be the same for all $d$; that is, $M_{d}=m_{d}$ for all $d$. Moreover, $M_{d+1}=M_{d}-k$ if and only if there are exactly $k$ roots of the Hilbert polynomial between $d-M_{d}$ and $d+1-M_{d+1}$. By induction we see that the value of $M_{d}$ is equal to the number of roots above $d$.

The condition of natural cohomology implies that the value of $|\chi(\mathcal{E}(d))|$ is the value of some $h^{j} \mathcal{E}(d)$. The formulas above tell us the value of $j$. The zeros determine the Hilbert polynomial as $\chi(\mathcal{E}(d))=C \cdot \prod_{i=1}^{s}\left(d-z_{i}\right)$ for some constant $C$, and $C$ can be computed by comparing leading coefficients, yielding the formula given.

Remark 6.5. A coherent sheaf $\mathcal{E}$ on $\mathbb{P}^{m}$ is a vector bundle if and only if the intermediate cohomology modules $\bigoplus_{d} H^{i} \mathcal{E}(d)$ for $1 \leq i \leq m-1$ have finite length. Thus a supernatural sheaf on $\mathbb{P}^{m}$ of full dimension $\operatorname{dim} \mathcal{E}=m$ is a vector bundle by Theorem 6.4

The rank provided by Theorem 6.1 is not always the smallest possible. For example, the root sequence $z=(2,0,-2)$ occurs for any null-correlation bundle on 
$\mathbb{P}^{3}$ with monad

$$
0 \rightarrow \mathcal{O}_{\mathbb{P}^{3}}(-3) \rightarrow \mathcal{O}_{\mathbb{P}^{3}}^{4}(-2) \rightarrow \mathcal{O}_{\mathbb{P}^{3}}(-1) \rightarrow 0 .
$$

Such a bundle has rank 2 , while Theorem 6.4 provides a bundle of rank 6 . A general lower bound for the rank, which gives 2 in this case, is provided by the following:

Proposition 6.6. Let $z=\left(z_{1}>\cdots>z_{m}\right)$ be a root sequence for supernatural bundles $\mathcal{E}$ on $\mathbb{P}^{m}$. For each prime $p$ let $e_{p}(z)$ denote the maximal integer such that the root sequence $z$ contains each residue $\bmod p$ at least $e_{p}(z)$ times, and let $c(z)=\prod_{p} p^{e_{p}(z)}$. Then the rank of a supernatural bundle with root sequence $z$ is a multiple of $\frac{m !}{c(z)}$.

Proof. The polynomial

$$
\frac{1}{c(z)} \prod_{1}^{m}\left(t-z_{j}\right)
$$

takes integral values which have no common factor.

Remark 6.7. Note that $\operatorname{rank} \frac{m !}{c(z)}$ is not always possible for a supernatural bundle with root sequence $z$. A simple example is the root sequence $(2,1,-2,-3)$, which is not possible for a rank 2 or even rank 4 vector bundle on $\mathbb{P}^{4}$. In fact, the Tate resolution for a supernatural bundle of rank $2 r$ with this root sequence would be

$\begin{array}{cccccccc}56 r & 21 r & 5 r & 0 & 0 & 0 & 0 & 0 \\ 0 & 0 & 0 & 0 & 0 & 0 & 0 & 0 \\ 0 & 0 & 0 & r & r & 0 & 0 & 0 \\ 0 & 0 & 0 & 0 & 0 & 0 & 0 & 0 \\ 0 & 0 & 0 & 0 & 0 & 5 r & 21 r & 56 r\end{array}$

However the entries of the $r \times r$ matrix of linear forms must span all the linear forms on $\mathbb{P}^{4}$, so $r \geq 3$. In fact, a generic choice of the $3 \times 3$ matrix leads to such bundles with rank 6 , which is the rank provided by the proof of Theorem 6.1. Moreover, Theorem 6.2 gives a bundle of this type having rank 20 so that, at least in characteristic zero, every sufficiently large even number occurs as the rank of a supernatural bundle with root sequence $(2,1,-2,-3)$.

For arbitrary root sequences $z$, we conjecture that any sufficiently high multiple of $\frac{m !}{c(z)}$ actually occurs as a rank.

Linear monads for vector bundles, given by the next proposition, will be central in our use of supernatural bundles.

Proposition 6.8. Let $\mathcal{E}$ be a vector bundle on $\mathbb{P}^{m}$, and let a be an integer. If $\mathcal{E}^{*}$ is a-regular, then there exists a linear complex

$$
0 \rightarrow E^{0} \rightarrow E^{1} \rightarrow \cdots \rightarrow E^{m} \rightarrow 0
$$

with $E^{k}=S(a+k)^{b_{k}}$, with homology $H^{i} E=\sum_{d} H^{i} \mathcal{E}(d)$ for $i<m$ and $H^{m} E=$ $\sum_{d \geq-a-m} H^{m} \mathcal{E}(d)$.

Proof. Since $\mathcal{E}^{*}$ is a-regular, $\sum_{d \geq a} H^{0} \mathcal{E}^{*}(d)$ has a linear resolution

$$
0 \leftarrow \sum_{d \geq a} H^{0} \mathcal{E}^{*}(d) \leftarrow S(-a)^{b_{0}} \leftarrow \cdots \leftarrow S(-a-m)^{b_{m}} \leftarrow 0 .
$$

The dual complex is the desired complex $E$. Its sheafification $\tilde{E}$ has homology $H^{0}(\tilde{E}) \cong \mathcal{E}$ and is exact otherwise. The statement about the homology follows by chasing sheaf cohomology through the sheafified complex. See Section 8 of Eisenbud-Fløystad-Schreyer [2003] for more about linear monads. 
Proposition 6.8 applies to vector bundles with supernatural cohomology:

Proposition 6.9. Let $\mathcal{E}$ on $\mathbb{P}^{m}$ be a vector bundle with supernatural cohomology with zeroes $z_{1}>\cdots>z_{m}$ of the Hilbert polynomial. Let $a \geq-z_{m}-m$ be an integer. Then $\mathcal{E}^{*}$ is a-regular, and the complex constructed from $\mathcal{E}$ as in Proposition 6.8 has supernatural cohomology.

Proof. By Theorem 6.4 the dual bundle $\mathcal{E}^{*}$ is $a$-regular. Indeed,

$$
h^{i} \mathcal{E}^{*}(a-i)=h^{m-i} \mathcal{E}(i-a-m-1)=0 \text { for } i \geq 1,
$$

because $i-a-m-1 \leq i-1+z_{m-1} \leq z_{m-i}$.

\section{Proof of the Boij-Söderberg Conjectures}

Both Theorems 0.1 and 0.2 reduce at once to the case $c=n$ of modules of finite length. To simplify notation we will work in that context.

Theorem 7.1. The cone defined by the upper facet equations contains the Betti tables of minimal free resolutions of all finitely generated graded $S$-modules.

Proof. Suppose $f=\left(f_{0}<f_{1}<\cdots<f_{n}\right)$ is a degree sequence and $\tau$ is an integer with $0 \leq \tau \leq n-1$ such that $f_{\tau+1}=f_{\tau}+2$, so that facet $(f, \tau)$ is defined. By Theorem 6.1 there is a vector bundle $\mathcal{E}$ on $\mathbb{P}^{n-1}$ with supernatural cohomology whose Hilbert polynomial $\chi(\mathcal{E}(d))$ has roots

$$
\left(z_{1}>\cdots>z_{n-1}\right)=\left(-f_{0}>\cdots>-f_{\tau-1}>-f_{\tau+2}>\cdots>-f_{n}\right) .
$$

Let

$$
E: \quad 0 \rightarrow E^{0} \rightarrow \cdots \rightarrow E^{m} \rightarrow 0
$$

be the linear complex made from $\mathcal{E}$ with $a=f_{n}-n+1$ as in Proposition 6.8, so that $E^{0}=S(a)^{b_{0}}$. Set $c=f_{\tau}$. Theorem 4.1 proves that $\langle-, E\rangle_{c, \tau}$ is non-negative on the cone of Betti tables of all minimal resolutions. Thus it suffices to prove that facet $(f, \tau)$ is defined by the vanishing of this functional.

By construction, the coefficient of $\beta_{i, j}$ for $j \geq f_{i}^{+}$in $\langle-, E\rangle_{c, \tau}$ is zero, so $\langle F, E\rangle_{c, \tau}=0$ for all resolutions $F$ such that $\beta_{i, j}(F)=0$ for all $i, j$ with $j<f_{i}^{+}$. On the other hand we have

$$
\langle F, E\rangle_{c, \tau}=\langle F, E\rangle
$$

for a resolution $F$ of a module $M$ such that $\beta_{i, j}(M)=0$ for all $i, j$ with $j>f_{i}^{-}$. For these modules we check the vanishing criterion of Theorem 3.1. We have

$$
\operatorname{reg} M \leq f_{n}-n \text { and } \operatorname{reg} E_{0}=-f_{n}+n-1,
$$

so the first inequality in Theorem 3.1 is satisfied. For the remaining inequality, note that if $j>0$, then

$$
\operatorname{reg} F_{j-1} \leq f_{j-1} \text { and } \operatorname{reg} H^{j}(E)=z_{j-1}-1 .
$$

Thus $\langle-, E\rangle_{c, \tau}$ vanishes on $\operatorname{facet}(f, \tau)$. Finally we observe that $\langle F, E\rangle \neq 0$ for the pure complex $F$ with degree sequence $f$. We conclude that $\langle-, E\rangle_{c, \tau}$ is the supporting equation of this facet.

Proof of Theorem 0.2. As discussed in $₫ 2$ the Boij-Söderberg fan is simplicial, so every Betti table in it is in a unique minimal cone defined by a chain of degree sequences. The result follows from Theorem 7.1 
We return to the Example 2.4 of facet $((-4,-3,0,2,4,6,7,9), 3)$. The equation of the supporting hyperplane, computed before, is given by the dot product with the matrix

$\begin{array}{ccccccccc}\vdots & \vdots & \vdots & \vdots & \vdots & \vdots & \vdots & \vdots & \\ 1755 & -385 & 0 & 0 & 66 & -70 & 0 & 100 & \\ 385 & 0 & 0 & -66 & 70 & 0 & -100 & 175 & \\ 0^{*} & 0^{*} & 66 & -70 & 0 & 100 & -175 & 189 & \\ 0 & 0 & 70 & 0 & -100 & 175 & -189 & 140 & \\ 0 & 0 & 0^{*} & 100 & -175 & 189 & -140 & 60 & \\ 0 & 0 & 0 & 175 & -189 & 140 & -60 & 0 & \text { 0-th row } \\ 0 & 0 & 0 & 0^{+} & 0^{+} & 60 & 0 & 0 & \\ 0 & 0 & 0 & 0 & 0 & 0^{*} & 0^{*} & 44 & \\ 0 & 0 & 0 & 0 & 0 & 0 & 0 & 0^{*} & \\ 0 & 0 & 0 & 0 & 0 & 0 & 0 & 0 & \\ \vdots & \vdots & \vdots & \vdots & \vdots & \vdots & \vdots & \vdots & \end{array}$

Now we can interpret all the entries of this table: Suppose there is vector bundle $\mathcal{E}$ on $\mathbb{P}^{6}$ with natural cohomology for $\left(z_{1}>\cdots>z_{6}\right)=(4,3,0,-6,-7,-9)$. The coefficients of the facet equation, up to a common factor, are the dimensions of cohomology groups of $\mathcal{E}$. The rank of the bundle $\mathcal{E}$ is at least 15 by Proposition 6.6. and if such a bundle of rank 15 exists, then the integral factor above is 1 . In this case the monad $E$ for $\mathcal{E}$ would have shape

$$
0 \rightarrow S(3)^{455} \rightarrow S(4)^{1260} \rightarrow S(5)^{1480} \rightarrow S(6)^{924} \rightarrow S(7)^{308} \rightarrow S(8)^{44} \rightarrow 0
$$

with homology $H^{i}(E)$ only in (cohomological) degrees $i=0,2,3,5$. Indeed the Hilbert series $H_{E}(t)$ of such a complex would be

$$
\begin{aligned}
H_{E}(t)= & \left(-44 t^{-8}+308 t^{-7}-924 t^{-6}+1480 t^{-5}-1260 t^{-4}+455 t^{-3}\right) /(1-t)^{7} \\
= & -\left(44 t^{-8}\right)-\left(60 t^{-5}+140 t^{-4}+189 t^{-3}+175 t^{-2}+100 t^{-1}\right) \\
& +\left(70 t^{1}+66 t^{2}\right)+\left(385 t^{5}+1755 t^{6}+\cdots\right) .
\end{aligned}
$$

We do not know whether there is such a bundle with rank as small as 15 . The bundle provided by Theorem 6.1 has rank $\left(\begin{array}{c}6 \\ 2,1,2,1\end{array}\right)=180=12 \times 15$. The complex $E$ that corresponds to it thus has Betti numbers 12 times larger than the hypothetical monad indicated above. The homogeneous bundle of Theorem 6.2 has rank $3^{5} \times 5 \times$ $7 \times 11 \times 13=1216215$ in this case. Hence in characteristic zero, any sufficiently large multiple of $3 \times 15=45$ occurs as a rank for the root sequence $(4,3,0,-6,-7,-9)$. In Remark 6.7 we conjectured that any sufficiently high multiple of 15 occurs as a rank.

\section{The CONE OF COHOMOlogy tables}

Throughout this section we set $m=n-1$ and consider coherent sheaves and vector bundles on $\mathbb{P}^{m}=\mathbb{P}_{K}^{m}$.

Let $\mathcal{E}$ be a vector bundle on $\mathbb{P}^{m}$. By Serre vanishing and Serre duality only the 0 -th and $m$-th row of the cohomology table of $\mathcal{E}$ can have infinitely many non-zero entries. We define the cohomology range

$$
\begin{aligned}
r(\mathcal{E}) & =\left(r_{1}(\mathcal{E}) \geq \cdots \geq r_{m+1}(\mathcal{E})=-\infty\right), \\
R(\mathcal{E}) & =\left(\infty=R_{0}(\mathcal{E}) \geq \cdots \geq R_{m}(\mathcal{E})\right)
\end{aligned}
$$

of $\mathcal{E}$ by the formulas

$$
\begin{aligned}
r_{i}(\mathcal{E}) & =\sup \left\{d \mid H^{j} \mathcal{E}(d-j)=0 \text { for all } j<i\right\}, \\
R_{i}(\mathcal{E}) & =\inf \left\{d \mid H^{j} \mathcal{E}(d-j)=0 \text { for all } j \geq i\right\} .
\end{aligned}
$$


For example, $R_{1}(\mathcal{E})$ is the Castelnuovo-Mumford regularity of $\mathcal{E}$. It follows from the definition that $h^{i} \mathcal{E}(d-i)=0$ except possibly when $r_{i+1}(\mathcal{E})<d<R_{i}(\mathcal{E})$, whence the name "cohomology range".

Proposition 8.1. If $\mathcal{E}$ is a supernatural vector bundle on $\mathbb{P}^{m}$, then

$$
\max \left\{i \mid h^{i} \mathcal{E}(d-i) \neq 0\right\}=\min \left\{i \mid h^{i} \mathcal{E}(d-i) \neq 0\right\}
$$

and

$$
r_{i}(\mathcal{E})=R_{i}(\mathcal{E})=z_{i}(\mathcal{E})+i .
$$

Proof. Immediate from Theorem 6.4

We now define the fan of supernatural cohomology tables inside $\mathbb{B}^{*}$. We work with root sequences $z$ of a given length $m=n-1$. First, we define the supernatural cohomology table of the root sequence $z=\left(z_{1}>\cdots>z_{m}\right)$ by the formula

$$
\gamma_{i, d}(z)= \begin{cases}\frac{1}{m !} \prod_{j=0}^{m-1}\left|d-z_{j}\right| & \text { for } z_{i}>d>z_{i+1}, \\ 0 & \text { otherwise }\end{cases}
$$

where we set $z_{0}=\infty$ and $z_{m+1}=-\infty$ for convenience. We partially order these supernatural cohomology tables by the termwise partial order on their root sequences

$$
z=\left(z_{1}>\cdots>z_{m}\right) \geq z^{\prime}=\left(z_{1}^{\prime}>\cdots>z_{m}^{\prime}\right) \Leftrightarrow z_{i} \geq z_{i}^{\prime} \text { for all } i .
$$

This coincides with the other obvious way of defining a partial order:

Proposition 8.2. If $\mathcal{E}$ and $\mathcal{E}^{\prime}$ are supernatural bundles, then $z(\mathcal{E}) \geq z\left(\mathcal{E}^{\prime}\right)$ if and only if

$$
\max \left\{i \mid h^{i} \mathcal{E}(d-i) \neq 0\right\} \geq \max \left\{i \mid h^{i} \mathcal{E}^{\prime}(d-i) \neq 0\right\} \text { for all } d .
$$

Proof. If $\mathcal{E}$ is supernatural, then $\max \left\{i \mid h^{i} \mathcal{E}(d-i) \neq 0\right\}=\min \left\{i \mid h^{i} \mathcal{E}(d-i) \neq 0\right\}$ by Proposition 8.1. The result follows.

In a bounded range of root sequences, say $z$ with $a \geq z_{1}>\cdots>z_{m} \geq b$, all maximal chains of root sequences have the same length. Hence the order complex of chains (= totally ordered subsets) is equidimensional. The simplices defined by a chain of supernatural cohomology tables intersect only in common faces. This follows by the same argument as for pure resolutions of Cohen-Macaulay modules given in Boij-Söderberg [2006, Proposition 2.9. Thus the supernatural cohomology tables of root sequences in a bounded range define a geometric realization of the order complex of such root sequences, and this defines the fan of cohomology tables. Theorem 0.5, which we will prove below, implies the convexity of this fan.

Much of Proposition 2.1 remains true for the fan of cohomology tables. However, the outer facets that are not defined by a single $\gamma_{i, d}$ correspond this time to a sequence $z^{+}>z>z^{-}$, which differ only in one position, say $i$, and $z_{i}^{+}-1=z_{i}=$ $z_{i}^{-}+1$. A consequence of Theorem 8.4 below is that the equation of the outer facet coincides for all maximal chains containing the sequence $z^{+}>z>z^{-}$.

Let $F$ be a minimal free resolution of a graded $S$-module of finite length. In the following we set

$$
\langle F, \mathcal{E}\rangle:=\langle F, E\rangle,
$$

where $E=E_{a}$ is the dual of the free resolution of $\bigoplus_{d \geq a} H^{0} \mathcal{E}^{*}(d)$ for $a \gg 0$ as in Proposition 6.8. In case of a pure resolution $F$ with degree sequence $f$ the formula 
simplifies to

$$
\langle F, \mathcal{E}\rangle=\sum_{j \leq i}(-1)^{i-j} \beta_{i, f_{i}} h^{j}\left(\mathcal{E}\left(-f_{i}\right)\right) .
$$

Example 8.3. The non-zero cohomology groups for the three root sequences $z^{+}=(3,1,-4), z=(3,0,-4)$ and $z^{-}=(3,-1,-4)$ are indicated in the following diagram:

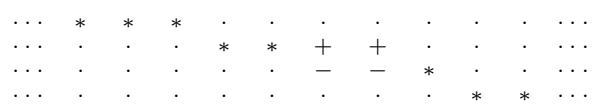

We can find the equation for the supporting hyperplane of a facet using an algorithm that is completely analogous to that of Proposition 2.2. Applying it in our example, we obtain the linear form whose coefficients are indicated in the following table:

$$
\begin{array}{cccccccccccc}
\ldots & 0^{*} & 0^{*} & 0^{*} & 0 & 0 & 0 & 0 & 0 & 0 & 0 & \ldots \\
\ldots & 0 & 0 & 2 & 0^{*} & 0^{*} & 0^{+} & 0^{+} & 0 & 0 & 0 & \ldots \\
\ldots & 0 & -2 & 0 & 0 & 35 & -70 & 42 & 0^{*} & 0 & 0 & \ldots \\
\ldots & 2 & 0 & 0 & -35 & 70 & -42 & 0 & 5 & 0^{*} & 0^{*} & \ldots
\end{array}
$$

The shape of the facet equation leads us to consider a pure complex

$$
F: 0 \rightarrow S^{2}(-4) \rightarrow S^{35}(-1) \rightarrow S^{70} \rightarrow S^{42}(1) \rightarrow S^{5}(3) \rightarrow 0,
$$

corresponding to the degree sequence $f$ that is the negative of the union of the root sequences $z^{+}, z$ and $z^{-}$.

The coefficients of $\langle F,-\rangle$ are

$$
\begin{array}{cccccccccccc}
\ldots & 0^{*} & 0^{*} & 0^{*} & -2 & 0 & 0 & 35 & 0 & 0 & & \ldots \\
\ldots & 0 & 0 & 2 & 0^{*} & 0^{*} & -35 & 70 & 0 & 0 & 0 & \ldots \\
\ldots & 0 & -2 & 0 & 0 & 35 & -70 & 42 & 0^{*} & 0 & 0 & \ldots \\
\ldots & 2 & 0 & 0 & -35 & 70 & -42 & 0 & 5 & 0^{*} & 0^{*} & \ldots
\end{array}
$$

These coefficients coincide with those of the facet equation except that in the facet equation some terms in the top two rows have been replaced by zeros. This is exactly the effect of the modification described in 44 .

Theorem 8.4. Suppose that the three root sequences $z^{+}>z>z^{-}$differ only in the $i$-th spot, where they have $z_{i}^{+}-1=z_{i}=z_{i}^{-}+1$. Let $f$ be the degree sequence that is the negative of the union of these three root sequences, and let $\tau$ be such that $f_{\tau}=-z_{i}$, the middle value. Let $c=f_{\tau-1}=f_{\tau}-1$, the smaller value. Let $F$ be a pure resolution corresponding to the degree sequence $f$. The functional $\langle F, \mathcal{E}\rangle_{c, \tau}$ is positive on the supernatural bundle $\mathcal{E}$ with root sequence $z$, and vanishes on all supernatural bundles $\mathcal{E}$ with root sequence $\leq z^{-}$or $\geq z^{+}$.

Proof. The functional coming from a pure resolution $F$ with degree sequence $f$ is

$$
\begin{aligned}
\langle F, \mathcal{E}\rangle_{c, \tau} & =\sum_{j=i<\tau}(-1)^{i-j} \beta_{i, f_{i}} h^{j} \mathcal{E}\left(-f_{i}\right) \\
& +\sum_{j=i-1<\tau}(-1)^{i-j} \beta_{i, f_{i}} h^{j} \mathcal{E}\left(-f_{i}\right) \\
& +\sum_{j \leq i-2}^{j-j}(-1)^{i-j} \beta_{i, f_{i}} h^{j} \mathcal{E}\left(-f_{i}\right) .
\end{aligned}
$$

It vanishes trivially on all supernatural bundles with degree sequence $\geq z^{+}$and is positive on the one with root sequence $z$ because the coefficients of the diagonal terms $i=j$ are positive. If $\mathcal{E}$ is a supernatural bundle with root sequence $\leq z^{-}$, then 
$\langle F, \mathcal{E}\rangle_{c, \tau}=\langle F, \mathcal{E}\rangle$. To prove that this is zero, we check the conditions of Theorem 3.1 .

The module $M=\operatorname{coker}\left(F_{1} \rightarrow F_{0}\right)$ has regularity $f_{n}-n$. The module $E^{0}$ has regularity $\operatorname{reg} E^{0}=z_{m}(\mathcal{E})+m=w_{m}+m \leq z_{m}^{-}+m \leq z_{m}^{+}+m=-f_{n}+m$. Thus

$$
\operatorname{reg} M+\operatorname{reg} E^{0} \leq f_{n}-n-f_{n}+m=-1<0 \text {. }
$$

Moreover for $j>0$, we have $\operatorname{reg} H^{j}(E)=w_{j}-1 \leq z_{j}^{-}-1 \leq-f_{j-1}-1$ and $\operatorname{reg} F_{j-1}=f_{j-1}$, hence

$$
\operatorname{reg} F_{j-1}+\operatorname{reg} H^{j}(E) \leq-1<0 .
$$

Proof of Theorem 0.5 . By Theorem 8.4 the equation of the non-trivial outer facets are given by functionals $\langle F,-\rangle_{c, \tau}$ for suitable pure free resolutions $F$ and integers $c, \tau$. By Theorem 4.1, this functional is non-negative on the monad $E=E_{a}$ obtained from the free resolution of $\bigoplus_{d \geq a} H^{0} \mathcal{E}^{*}(d)$ with $a \gg 0$ for any vector bundle $\mathcal{E}$.

Proof of Corollary 0.6. By restriction to a hyperplane and induction we see that the Hilbert polynomial of $\mathcal{E}$ has the form

$$
p_{\mathcal{E}}(t)=(\operatorname{rank} \mathcal{E})\left(\begin{array}{c}
t+m \\
m
\end{array}\right)+\operatorname{deg} \mathcal{E}\left(\begin{array}{c}
t+m-1 \\
m-1
\end{array}\right)+O\left(t^{m-2}\right)
$$

Thus

$$
\frac{p_{\mathcal{E}}(t)}{\operatorname{rank} \mathcal{E}}=\frac{t^{m}}{m !}+\left(\frac{1}{m !}\left(\begin{array}{c}
m+1 \\
2
\end{array}\right)+\frac{\mu(\mathcal{E})}{(m-1) !}\right) t^{m-1}+O\left(t^{m-2}\right),
$$

and in particular

$$
\mu(\mathcal{E})=-\frac{1}{m} \sum_{i=1}^{m}\left(z_{i}+i\right)
$$

where $z_{1}, \ldots, z_{m}$ are the roots of $p_{\mathcal{E}}$.

The cohomology table of $\mathcal{E}$ is a rational combination of cohomology tables of vector bundles $\mathcal{E}_{k}$ with supernatural cohomology. Hence the normalized Hilbert polynomial of $\mathcal{E}$ may be written in the form

$$
\frac{p_{\mathcal{E}}(t)}{\operatorname{rank} \mathcal{E}}=\sum_{k} \lambda_{k} \frac{p_{\mathcal{E}_{k}}(t)}{\operatorname{rank} \mathcal{E}_{k}}
$$

for some $\lambda_{k}>0$. Comparing coefficients of $t^{m}$ and $t^{m-1}$ we obtain $\sum_{k} \lambda_{k}=1$ and $\sum_{k} \lambda_{k} \mu\left(\mathcal{E}_{k}\right)=\mu(\mathcal{E})$.

On the other hand, by Theorem 6.4 the zeroes of the $\mathcal{E}_{k}$ satisfy

$$
r_{i}(\mathcal{E}) \leq r_{i}\left(\mathcal{E}_{k}\right)=z_{i}\left(\mathcal{E}_{k}\right)+i=R_{i}\left(\mathcal{E}_{k}\right) \leq R_{i}(\mathcal{E}),
$$

because otherwise $\mathcal{E}_{k}$ would contribute to a cohomology group outside the range of $\mathcal{E}$. From the expression for $\mu\left(\mathcal{E}_{k}\right)$ in terms of $z_{i}\left(\mathcal{E}_{k}\right)$ we get

$$
\frac{1}{m} \sum_{i=1}^{m} r_{i}(\mathcal{E}) \leq-\mu\left(\mathcal{E}_{k}\right) \leq \frac{1}{m} \sum_{i=1}^{m} R_{i}(\mathcal{E}) \text {. }
$$

Multiplying this inequality by $\lambda_{k}$ and summing over $k$ we obtain the assertion of the corollary. 


\section{ACKNOWLEDGEMENTS}

We are grateful to Jerzy Weyman for showing us that Ulrich Sheaves could be constructed from homogeneous bundles and teaching us about their cohomology; and to Dan Grayson and Mike Stillman, for their tireless assistance with Macaulay 2. We would never have discovered the crucial ideas in 93 and 94 without the extensive family of examples provided by their program M2. We also thank Daniel Erman and Steven Sam for interesting discussions and computer experiments related to this paper, and Silvio Levy for his expert and unstinting help with TeX. A group of young mathematicians at the 2008 AMS Math Research Communities meeting in Snowbird, Utah, read this paper carefully and made many suggestions about its exposition, which we have gratefully adopted.

\section{REFERENCES}

[2006] M. Boij and J. Söderberg. Graded Betti numbers of Cohen-Macaulay modules and the multiplicity conjecture. math.AC/0611081.

[2008] M. Boij and J. Söderberg. Betti numbers of graded modules and the Multiplicity Conjecture in the non-Cohen-Macaulay case. Preprint: arXiv:0803.1645.

[1973a] D. Buchsbaum and D. Eisenbud. Remarks on ideals and resolutions. Symposia Math. XI (1973) 193-204. MR0337946 (49:2715)

[1973b] D. A. Buchsbaum and D. Eisenbud. What makes a complex exact? J. Algebra 25 (1973) 259-268. MR0314819 (47:3369)

[1995] D. Eisenbud. Commutative Algebra with a View Toward Algebraic Geometry. Graduate Texts in Math. 150, Springer-Verlag, New York (1995). MR1322960|(97a:13001)

[2003] D. Eisenbud, G. Fløystad and F.-O. Schreyer. Sheaf cohomology and free resolutions over exterior algebras. Trans. Amer. Math. Soc. 355 (2003) 4397-4426. MR 1990756 (2004f:14031)

[2007] D. Eisenbud, G. Fløystad and J. Weyman. The existence of pure free resolutions. arXiv:0709.1529.

[2003] D. Eisenbud and F.-O. Schreyer. Resultants and Chow forms via exterior syzygies. J. Amer. Math. Soc. 16 (2003) 537-579. MR1969204 (2004j:14067)

[2007] D. Erman. The Semigroup of Betti Diagrams. arXiv:0806.4401

[2007] C.A Francisco and H. Srinivasan. Multiplicity conjectures. In Syzygies and Hilbert Functions, ed. I. Peeva, Lect. Notes in Pure and Appl. Math., Chapman and Hall, NY, 2007. MR2309929 (2008f:13044)

[M2] D. R. Grayson and M. E. Stillman. Macaulay 2, a software system for research in algebraic geometry. Available at http://www.math.uiuc.edu/Macaulay2/

[1982] R. Hartshorne and A. Hirschowitz. Cohomology of a general instanton bundle. Ann. Sci. de l'École Normale Sup. 15 (1982) 365-390. MR683638 (84c:14011)

[1984] J. Herzog and M. Kühl. On the Betti numbers of finite pure and linear resolutions. Comm. in Alg. 12 (13) (1984) 1627-1646. MR:743307 (85e:13021)

[1998] J. Herzog and H. Srinivasan. Bounds for multiplicities. Trans. Am. Math. Soc. 350 (1998) 2879-2902. MR1458304 (99g:13033)

[1974] D. Kirby. A sequence of complexes associated to a matrix. J. London Math. Soc. 7 (1974) 523-530. MR0337939 (49:2708)

[2008] M. Kunte. Gorenstein modules of finite length. Thesis, Uni. des Saarlandes (2008).

[1992] S. Mukai. Curves and symmetric spaces. Proc. Japan Acad. Ser. A Math. Sci. 68 (1992), no. 1, 7-10. MR.1158012 (93d:14042)

[2003] S. Mukai. Curves and Symmetric Spaces II. RIMS preprint 2003, http://www.kurims. kyoto-u.ac.jp/preprint/preprint_y2003.html

[1973] C. Peskine and L. Szpiro. Dimension projective finie et cohomologie locale. Applications la démonstration de conjectures de M. Auslander, H. Bass et A. Grothendieck. Inst. Hautes Etudes Sci. Publ. Math. 42 (1973) 47-119. MR0374130(51:10330)

[1986] F.-O. Schreyer. Syzygies of canonical curves and special linear series. Math. Ann. 275 (1986), no. 1, 105-137. MR849058 (87j:14052) 
[2006] J. Söderberg. Graded Betti numbers and $h$-vectors of level modules. Preprint, arxiv:math.AC/0612047.

[2003] J. Weyman. Cohomology of Vector Bundles and Syzygies. Cambridge Tracts in Math. 149 (2003). MR1988690(2004d:13020)

Department of Mathematics, University of California, Berkeley, Berkeley, CaliFORNIA 94720

E-mail address: eisenbud@math.berkeley.edu

Mathematik und Informatik, Universität des Saarlandes, Campus E2 4, D-66123 SaARBRÜCKEn, Germany

E-mail address: schreyer@math.uni-sb.de 